\title{
NON-CONVEXITY OF THE OPTIMAL EXERCISE BOUNDARY FOR AN AMERICAN PUT OPTION ON A DIVIDEND-PAYING ASSET
}

\author{
by \\ Huibin Cheng \\ M.A., University of Pittsburgh, 2007
}

Submitted to the Graduate Faculty of

the Department of Mathematics in partial fulfillment

of the requirements for the degree of

Doctor of Philosophy

University of Pittsburgh

2011 


\section{UNIVERSITY OF PITTSBURGH \\ MATHEMATICS DEPARTMENT}

This dissertation was presented

by

\section{Huibin Cheng}

It was defended on

May 11th, 2011

and approved by

John Chadam, Mathematics Department

Xinfu Chen, Mathematics Department

Juan J. Manfredi, Mathematics Department

William J. Layton, Mathematics Department

Jean-Francois Richard, Economics Department

Dissertation Advisors: John Chadam, Mathematics Department,

Xinfu Chen, Mathematics Department 


\title{
NON-CONVEXITY OF THE OPTIMAL EXERCISE BOUNDARY FOR AN AMERICAN PUT OPTION ON A DIVIDEND-PAYING ASSET
}

\author{
Huibin Cheng, $\mathrm{PhD}$ \\ University of Pittsburgh, 2011
}

\begin{abstract}
In this thesis, we prove that the optimal exercise boundary of the American put option is not convex when the dividend rate of the underlying asset which follows a geometric Brownian motion, is slightly larger than the risk-free interest rate. We show that the non-convex region occurs very near the expiry time. Numerical evidence is also provided which suggests that the convexity of the optimal exercise boundary is restored when the dividend rate is sufficiently larger than the interest rate. In addition we provide the near-expiry and far-from-expiry behavior of the boundary. To complete the rigorous proofs, we also show that the optimal exercise boundary has $C^{\infty}$ regularity.
\end{abstract}




\section{TABLE OF CONTENTS}

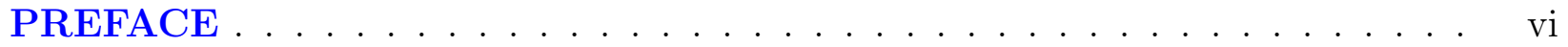

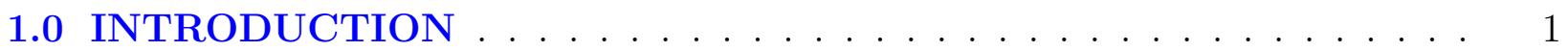

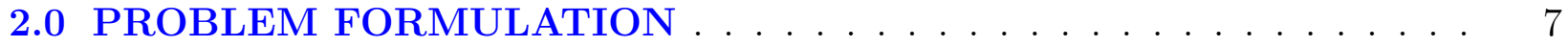

2.1 Put-call symmetry . . . . . . . . . . . . . . . . . 7

2.2 The Change of Variables . . . . . . . . . . . . . . . . . 8

3.0 WELL-POSEDNESS AND REGULARITY . . . . . . . . . . . . . 9

3.1 Basic Properties of the Solution . . . . . . . . . . . . . . . . . 9

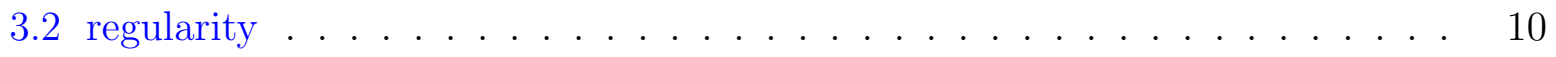

4.0 INTEGRAL EQUATIONS . . . . . . . . . . . . . . . . . . . . . 20

5.0 NUMERICAL SIMULATION . . . . . . . . . . . . . . . . . . . . 24

5.1 Numerical Scheme . . . . . . . . . . . . . . . . . . . . . . . 24

5.2 Numerical Results . . . . . . . . . . . . . . . . . . . . . . . . . . . 28

6.0 NON-CONVEXITY . . . . . . . . . . . . . . . . . . . . . . . 32

7.0 NEAR-EXPIRY BEHAVIOR . . . . . . . . . . . . . . . . . . 41

8.0 FAR-FROM EXPIRY BEHAVIOR . . . . . . . . . . . . . . . 46

9.0 CONCLUSIONS AND FUTURE WORK . . . . . . . . . . . . . 60

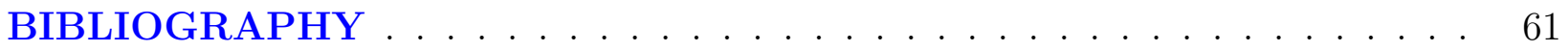




\section{LIST OF FIGURES}

1 Optimal time to exercise and optimal exercise boundary . . . . . . . . . 2

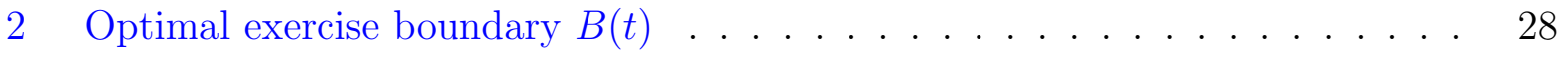

3 Loss of convexity as $\varepsilon:=\ln (D / r)$ crosses zero. . . . . . . . . . . . . 29

4 Interval for which the free boundary is not convex . . . . . . . . . 30

5 Interval for which the free boundary is not convex . . . . . . . . . . . 31 


\section{PREFACE}

The completion of my dissertation and subsequent Ph.D. has been a long journey. First and foremost I wish to express my deepest gratitude to my co-advisors Professor John Chadam and Professor Xinfu Chen for motivating my research interest in financial mathematics, sharing their incomparably profound knowledge with me, and patiently guiding my research. Without their enormous contributions of time and ideas, this thesis would not have been possible.

My thanks also go to the Department of Mathematics, University of Pittsburgh for financially supporting me. I would like to thank faculty in the department for my graduate study and friends for their enormous kind help and supports. Special thanks to the graduate student members of our financial mathematics group who have provided friendship as well as good advice and collaboration.

I am grateful to Professor William Layton, Professor Juan Manfredi, and Professor JeanFrancois Richard for serving on my thesis committee and providing insightful advice and comments.

Finally, I would like to thank my parents for their continuous support and my wife Mei Yang who was always there cheering me up and standing by me through good times and bad. 


\subsection{INTRODUCTION}

Options are traded both on exchanges and in the over-the-counter market. The options and derivatives market is significantly larger than the equity and bond markets combined. Moreover, the diversity and complexity of these financial instruments makes them difficult to accurately price and hedge. In this thesis we study some mathematical and numerical problems associated with pricing American put options with a geometric Brownian underlier. This is the prototypical example for studying option with early exercise capability.

An American put option is one exercise right of selling a share of underlying security (stock) at a fixed price called the strike, $E$, any time up to expiry, $T$. Black and Scholes theory can be used to derive the price (c.f. [42]) of the option, based on the following assumptions: (i) the stock price, $\mathbf{S}_{t}$, is a log-normal process, i.e.,

$$
d \mathbf{S}_{t}=\mu_{t} \mathbf{S}_{t} d t+\sigma \mathbf{S}_{t} d \mathbf{W}_{t},
$$

where $\sigma$ is a positive constant, $\left\{\mathbf{W}_{t}\right\}$ is the standard Brownian motion (Wiener process) and $\left\{\mu_{t}\right\}$ is an adapted process; (ii) for the time period $\left[t, t+d t\right.$ ), the stock pays $D \mathbf{S}_{t} d t$ dividend at time $t+d t$, where $D \geqslant 0$ is the constant dividend rate; (iii) there is a risk-free asset whose

return rate is a constant, $r$. The price of the option at time $t \leqslant T$ with observed stock price $S_{t}$ is $P\left(S_{t}, t\right)$ where $P(\cdot, \cdot)$ is the solution of the obstacle problem (variational inequality)

$$
\begin{cases}\max \left\{\mathcal{L}^{*} P,(E-S)^{+}-P\right\}=0 & \text { in }(0, \infty) \times(-\infty, T), \\ P(S, T)=(E-S)^{+}:=\max \{E-S, 0\} & \text { on }(0, \infty) \times\{T\} ;\end{cases}
$$

here $\mathcal{L}^{*}$ is the Black-Scholes operator defined by

$$
\mathcal{L}^{*} P=\frac{\partial P}{\partial t}+\frac{\sigma^{2}}{2} S^{2} \frac{\partial^{2} P}{\partial S^{2}}+(r-D) S \frac{\partial P}{\partial S}-r P .
$$


The option holder must decide on a strategy for the optimal time to exercise the position during the lifetime of the contract:

$$
T^{*}=\min \left\{t \in[0, T] \mid S_{t} \leqslant B(t)\right\}
$$

(c.f. Figure 1) i.e., the first time when the stock price drops to $B(t)$, where, the early

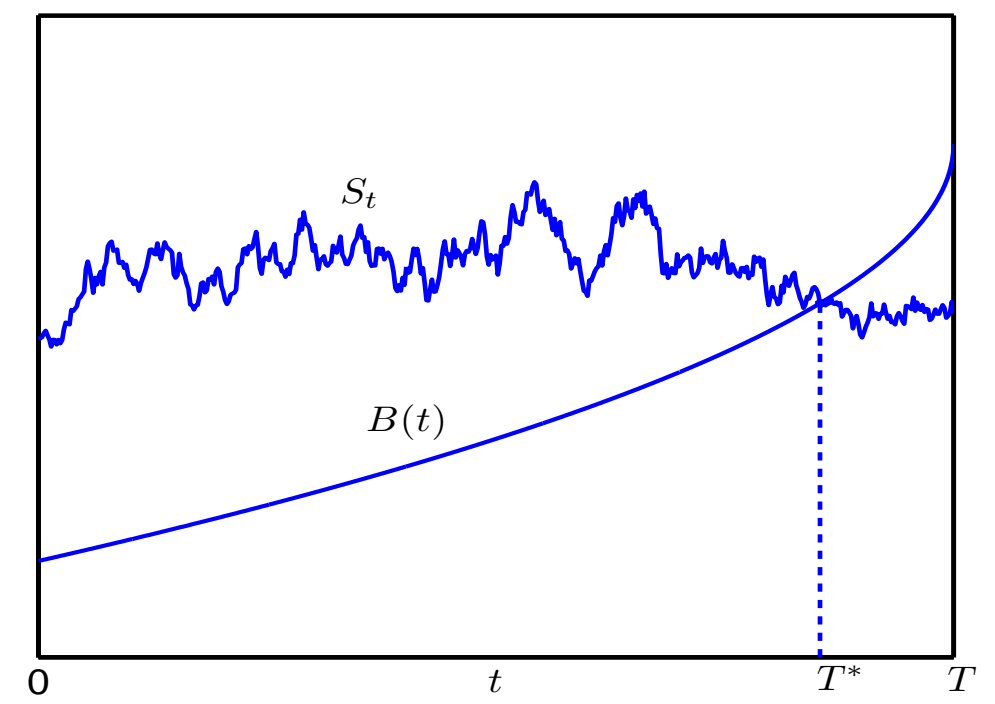

Figure 1: Optimal time to exercise and optimal exercise boundary

(optimal) exercise boundary $B(t)$ is defined in terms of the solution of (1.1) as

$$
B(t):=\inf \left\{S>0 \mid P(S, t)>(E-S)^{+}\right\} \quad \forall t \leqslant T
$$

The precise location of this boundary is of great importance to option traders. Using inaccurate estimates for the early exercise boundary could lead to large losses.

The American put option problem was first stated mathematically by McKean [37] and then studied by Moerbeke [39]. Since then it has been intensively studied in the mathematics and finance communities, see [2-7,9-13,15-18,23,27,30-34,36,40-42] and references therein. In contrast with European style options, that only allow exercise at expiry, the American options of interest here do not have closed form solutions, requiring numerical or analytic approximations for the price and the early exercise boundary. 
This thesis will focus on some mathematical open problems associated with the early exercise boundary. In particular, we will provide new rigorous results in three areas:

(i) the convexity and non-convexity of the early exercise boundary,

(ii) the $C^{\infty}$ regularity of the early exercise boundary, and

(iii) the behavior of the early exercise boundary near and far from expiry.

An integral part of this analytic and numerical study will be the derivation and use of several nonlinear integral equations for the free boundary.

The convexity of the early exercise boundary $B(t)$ becomes important for its global approximation and interpolation which is very useful for practioners. Recently, Chen, Chadam, Jiang and Zheng [17] provided a rigorous proof that the boundary $B(t)$ for the American put is convex when the dividend rate $D$ was zero; an independent proof was obtained by Ekstrom [18]. Since then, a great deal of effort has been made to show the convexity of the boundary for $D>0$. However, numerical experiments suggest that the early exercise boundary loses convexity when $D$ is slightly larger than $r$ and that convexity is maintained when $D \leq r$ and when $D$ is sufficiently larger than $r$.

We believe that this phenomenon is due to the following change of behavior of the early exercise boundary, $S=B(t)$, very near expiry, $T$, as $D$ increases past $r$. At expiry, the early exercise boundary approaches its expiry value with infinite velocity [39], leading to difficulties in the accurate approximation of numerical simulation and pricing in this extremely volatile period. Therefore, asymptotic expansions of the boundary near expiry had been studied earlier (see $[3,5,10,15,16,32,40,42]$ and references therein). As $t \nearrow T$, if $r>D, B(t)$ follows

$$
\ln \frac{B(t)}{E}=\sigma \sqrt{-(T-t) \ln \left[8 \pi(r-D)^{2}(T-t)\right]} .
$$

In the above expression, the correct constant was first found in [40], then is proven with mathematical rigor in [15]. If $r=D$, the $B(t)$ follows a similar behavior,

$$
\ln \frac{B(t)}{E}=\sigma \sqrt{-(T-t) \ln \left[4 \pi r^{2}(T-t)\right]} .
$$

As $D$ increases past $r$, the near expiry behavior of the boundary becomes quite different $[13,32]$, becoming

$$
\ln \frac{B(t)}{E}=\ln \frac{r}{D}-A_{0} \frac{\sigma}{\sqrt{2}} \sqrt{T-t}
$$


where the constant $A_{0}=0.90344 \ldots$ Therefore, for $D>r$ we have for

$$
B^{\prime}(t) \propto \frac{1}{\sqrt{T-t}}, \quad t \simeq T
$$

and the probability density of the associated Brownian motion, $\left[2 \pi \sigma^{2}(T-t)\right]^{-1 / 2} e^{-[B-B(T)]^{2} /\left[2 \sigma^{2}(T-t)\right]}$ is proportional to $B^{\prime}(t)$ in this regime. On the other hand, for $0 \leqslant D \leqslant r$, the dominating near-expiry behavior is

$$
B^{\prime}(t) \propto \frac{\sqrt{|\ln (T-t)|}}{\sqrt{T-t}},
$$

and the probability density is almost a positive constant. As a result, when $0<D-r \ll 1$, we can find times $t_{1}<T$, for the first behavior and, $t_{2}<t_{1}$, for the second behavior, such that $B(T-)=\infty>B^{\prime}\left(t_{2}\right)>B^{\prime}\left(t_{1}\right)>0$, thereby demonstrating that the free boundary is not convex. When $D-r$ is not small, it seems the second behavior is either suppressed or occurs too early so that the free boundary remains convex. Motivated by the above changing behaviors of $B(t)$ and the numerical results, we show that the convexity of $B(t)$ is lost when $0<D-r \ll 1$ and the non-convex region occurs near expiry through careful estimates obtained from a pair of integro-differential equations for the optimal exercise boundary.

Since these integral equations involve the derivative of the boundary, complete rigor relies on a proof of its regularity. Also the validity of the formulation (1.1) and (1.2) for the American put option relies on the following regularity of the solution of (1.1):

1. $P_{S}:=\partial P / \partial S$ is continuous,

2. $B$ is continuous, $P(S, t)>(E-S)^{+}$when $S>B(t)$, and $P(S, t)=(E-S)^{+}$when $S \leqslant B(t)$

Chen and Chadam [15] established the existence and uniqueness for the put option problem when the dividend payment is zero. The regularity of the early exercise boundary was recently investigated for more general underliers. Bayraktar and Xing [6] and Yang, Jiang and Bian [41] proved that the boundary is $C^{\infty}$ (except at maturity) when the stock price follows a jump-diffusion process and Lamberton and Mikow [33] showed its continuity for exponential Lévy process. Laurence and Salsa [35] proved $C^{\infty}$ regularity of the free boundary for an American option on several assets with convex payoff. These proofs are necessarily 
quite technical. In this thesis, we provide a self-contained short proof in the present log normal context that the optimal exercise boundary $S=B(t)$ of the American put option with dividend payment is $C^{\infty}$.

We anticipate the changing behaviors of $B(t)$ from our numerical results for general $D>0$ will require sharp estimates on the far-from-expiry (i.e., the scaled time-to-expiry $\left.s:=\frac{\sigma^{2}}{2}(T-t) \rightarrow \infty\right)$ behavior of the boundary $\left(B(t)=E e^{b(s)}\right.$; see section (2.2)). It is well known that $(p, b):=(P / E, \ln (B / E))$ approaches its Merton solution $\left(p^{*}, s^{*}\right)$ for the infinite horizon problem [38]. Recently Ahn, Choe and Lee [1] showed the following long time asymptotic behavior when $D=0$ :

$$
\begin{gathered}
b(s)=b^{*}+o(1) e^{-(k+1)^{2} s / 4}, \\
\left\|p(\cdot, s)-p^{*}(\cdot)\right\|_{L^{\infty}(\mathbb{R})}=O(1) \begin{cases}s^{-1 / 2} e^{-k s} & \text { if } 0<k<1, \\
e^{-(k+1)^{2} s / 4} & \text { if } k \geqslant 1,\end{cases}
\end{gathered}
$$

where $k=2 r \sigma^{-2}$. In [15] Chen and Chadam provided the outline of the proof of a stronger result:

$$
b(s)=b^{*}+[m+o(1)] s^{-3 / 2} e^{-(k+1)^{2} s / 4} \quad \text { as } s \rightarrow \infty,
$$

where $m$ is a positive constant that can be easily determined numerically. In this thesis we follow the steps outlined in [15] to provide a proof of our sharper result in the more general setting of a dividend-paying asset (i.e., for all dividend rates $D \geqslant 0$ ). We also provide generalizations of (1.7) for arbitrary $D \geqslant 0$. The precise statements of these results are given in chapter 8 . The proofs capture the changes in the estimates arising from the variation in $D$. Moreover, our proofs do not require the convexity of the free boundary in contrast with the results in [1] where it plays a crucial role. This observation is especially significant since the early exercise boundary is not convex when $0<D-r \ll 1$.

The thesis is organized as followed, in chapter 2, we begin by writing the American put option problem in a non-dimensional form. The connection between put and call options is also provided. In Chapter 3, we prove the regularity of $b(s)$. The integral equations required for the main result are derived in chapter 4 and in chapter 5 , the non-convexity of the optimal exercise boundary is proven when $0<D-r \ll 1$. In chapter 7 and 8 we provide 
the near-expiry and far-from expiry behaviors of $b(s)$ respectively. Finally, we conclude the thesis in chapter 9 with further research suggested by these results. 


\subsection{PROBLEM FORMULATION}

In this chapter, we derive the connection between American put and call options, then we reformulate our problem in non-dimensional form.

\section{$2.1 \quad$ PUT-CALL SYMMETRY}

The American put option problem is related to a corresponding problem for a American call option (see [32]). Define new variables

$$
\tilde{S}=\frac{E^{2}}{S}, \quad C(\tilde{S}, t)=\frac{E}{S} P(S, t),
$$

and constants $\tilde{r}=D, \tilde{D}=r$. Then inequality $P(S, t)>\max \{E-S, 0\}$ is equivalently to

$$
\frac{E \tilde{C}(\tilde{S}, t)}{\tilde{S}}>\max \left\{E-\frac{E^{2}}{\tilde{S}}\right\}, \quad \text { i.e., } \quad \tilde{C}(\tilde{S}, t)>\max \{\tilde{S}-E, 0\} \text {. }
$$

Direct computation yields

$$
\begin{aligned}
& \frac{\partial C}{\partial t}(\tilde{S}, t)=\frac{K}{S} \frac{\partial P}{\partial t}(S, t) ; \\
& \frac{\partial C}{\partial \tilde{S}}(\tilde{S}, t)=\frac{1}{E}\left(\frac{\partial P}{\partial S} S-P\right), \quad \frac{\partial^{2} C}{\partial \tilde{S}^{2}}(\tilde{S}, t)=-\frac{1}{E} \frac{\partial^{2} P}{\partial S^{2}} S^{3} .
\end{aligned}
$$

Substituting (2.1) and (2.2) into (1.1), one obtains the obstacle problem,

$$
\begin{cases}\max \left\{C_{t}+\frac{1}{2} \sigma^{2} \tilde{S}^{2} C_{\tilde{S} \tilde{S}}+(\tilde{r}-\tilde{D}) \tilde{S} C_{\tilde{S}}-\tilde{r} C,(\tilde{S}-E)^{+}-C\right\}=0 & \text { in }(0, \infty) \times(-\infty, T), \\ C(\tilde{S}, T)=(\tilde{S}-E)^{+}:=\max \{\tilde{S}-E, 0\} & \text { on }(0, \infty) \times\{T\} ;\end{cases}
$$


which is the variational inequality for the American call option with $C(\tilde{S}, t)$ being its option price when its underlying stock price is $\widetilde{S}$ at time $t$. Similar to the American put option problem, we can define the optimal exercise boundary $\tilde{B}(t)$ as

$$
\tilde{B}(t):=\sup \left\{\tilde{S}>0 \mid \tilde{C}(\tilde{S}, t)>(\tilde{S}-E)^{+}\right\} \quad \forall t \leqslant T .
$$

Thus the put option is transformed into the call through the identity,

$$
C\left(\frac{E^{2}}{S}, t ; D, r, \sigma, E, T\right)=\frac{S}{E} P(S, t ; r, D, \sigma, E, T), \quad \tilde{B}(t ; r, D)=\frac{E^{2}}{B(t ; D, r)}
$$

and hence it suffices to study the American put option.

\subsection{THE CHANGE OF VARIABLES}

It is mathematically convenient to use the dimensionless quantities:

$$
\begin{gathered}
x:=\ln \frac{S}{E}, \quad s:=\frac{\sigma^{2}}{2}(T-t), \quad k:=\frac{2 r}{\sigma^{2}}, \quad \ell:=\frac{2 D}{\sigma^{2}}, \quad \alpha:=k-\ell-1, \\
P(S, t)=E p(x, s)=E p\left(\ln \frac{S}{E}, \frac{\sigma^{2}}{2}(T-t)\right), \quad B(t)=E e^{b(s)} .
\end{gathered}
$$

In a typical financial situation we might have $\sigma=20 \% \quad\left(\right.$ year $\left.^{-1 / 2}\right), T-t=0.5$ (year), with $\mathbf{S}_{t}$ fluctuating in $(40 \% E, 250 \% E)$. The resulting range of interest for the dimensionless variable $(x, s)$ would be $x \in(-1,1), s \in[0,0.01]$.

With

$$
p_{0}(x):=\max \left\{1-e^{x}, 0\right\}, \quad \mathcal{L} p:=p_{x x}+\alpha p_{x}-k p,
$$

The variational form $(1.1)$ for $(B, P)$ is transformed to

$$
\max \left\{\mathcal{L} p-p_{s}, p_{0}-p\right\}=0 \quad \text { in } \mathbb{R} \times(0, \infty), \quad p(\cdot, 0)=p_{0}
$$

From the solution, we define

$$
b(s):=\inf \left\{x \mid p(x, s)>p_{0}(x)\right\} \quad \forall s>0, \quad b(0):=\lim _{s \searrow 0} b(s) .
$$

We shall call $x=b(s)$ the free boundary and use the default extension $b(0):=\lim _{s \searrow_{0}} b(s)$ and define $b_{0}:=\min \{0, \ln (k / l)\}\left(\right.$ when $\left.l=0, b_{0}=0\right)$. 


\subsection{WELL-POSEDNESS AND REGULARITY}

Using a penalty method, Chen and Chadam [15] showed that the problem (2.6) has a unique

solution for $l=0$ (i.e., $D=0) p \in C^{\beta, \beta / 2}(\mathbb{R} \times[0, \infty)) \cap W_{\gamma, l o c}^{2,1}\left(\mathbb{R} \times[0, \infty) \backslash\left[b_{0}, 0\right] \times\{0\}\right)$ for every $\gamma>1$ and $\beta \in(0,1)$. Moreover, $b(s)$ is a strictly decreasing continuous function on $(0, \infty)$ and $(p, b)$ solves the problem (3.3). For $l>0$, the proof is quite similar and omitted here. In this chapter we only consider the regularity of the free boundary and shall show that $b(s) \in C^{\infty}(0, \infty) \cap C[0, \infty)$.

\subsection{BASIC PROPERTIES OF THE SOLUTION}

In the next section we shall show that $b \in C^{\infty}((0, \infty))$, from which one can derive the following: for each $s>0$,

$$
\begin{gathered}
p(b(s), s)=1-e^{b(s)}, \\
p_{x}(b(s), s)=-e^{b(s)} \\
p_{s}(b(s), s)=0 \\
p_{x x}(b(s) \pm, s)=-e^{b(s)}+\left(\frac{1}{2} \pm \frac{1}{2}\right)\left(k-\ell e^{b(s)}\right), \\
p_{s x}(b(s) \pm, s)=\left(\frac{1}{2} \pm \frac{1}{2}\right) \dot{b}(s)\left(\ell e^{b(s)}-k\right),
\end{gathered}
$$

where \pm denotes limits from the right and left. The first and second equations are a direct consequence of $p(x, s)=p_{0}(x)=1-e^{x}$ for $x \leqslant b(s)$. Differentiating the equation $p(b(s), s)=$ $1-e^{b(s)}$ with respect to $s$ leads

$$
p_{s}(b(s), s)+p_{x}(b(s), s) \dot{b}(s)=-e^{b(s)} \dot{b}(s)
$$


which implies that $p_{s}(b(s), s)=0$. From $p_{s}(x, s)=\mathcal{L} p$ for $x>b(s)$, one obtains that

$$
p_{x x}(b(s)+, s)=p_{s}(b(s), s)-\alpha p_{x}(b(s), s)+k p(b(s), s)=-e^{b(s)}+\left(k-\ell e^{b(s)}\right) .
$$

The fact $p(x, s)=1-e^{x}$ for $x \leq b(s)$ implies that $p_{x x}(b(s)-, s)=-e^{b(s)}$. Therefore

$$
p_{x x}(b(s) \pm, s)=-e^{b(s)}+\left(\frac{1}{2} \pm \frac{1}{2}\right)\left(k-\ell e^{b(s)}\right)
$$

Similarly, differentiating $p_{x}(b(s) \pm, s)=p_{0 x}(b(s))$ we obtain the expression for $p_{s x}(b(s) \pm, s)$.

\subsection{REGULARITY}

Theorem 3.1. Problem (2.6) admits a unique solution, $p$. In addition, both $p_{x}$ and $p_{t}$ are continuous in $\mathbb{R} \times(0, \infty)$ and the function b defined in $(2.7)$ is in $C^{\infty}((0, \infty)) \cap C([0, \infty))$. Furthermore,

$$
b(0)=b_{0}, \quad p(x, s)>p_{0}(x) \forall x>b(s), \quad p(x, s)=p_{0}(x) \forall x \leqslant b(s) .
$$

As a result, $(p, b)$ is the solution of the free boundary problem

$$
\begin{cases}\mathcal{L} p-p_{s}=0>p_{0}-p & \text { in } Q_{b}:=\{(x, t) \mid t>0, x>b(t)\}, \\ p_{0}-p=0>\mathcal{L} p-p_{s} & \text { in }\{(x, t) \mid t>0, x<b(t)\}, \\ \lim _{x \preceq b(t)} p(x, t)=p_{0}(b(t)) & \forall t>0, \\ \lim _{x \searrow b(t)} p_{x}(x, t)=p_{0 x}(b(t)) & \forall t>0, \\ p(\cdot, 0)=p_{0}(\cdot) & \text { on } \mathbb{R} \times\{0\} .\end{cases}
$$


The problem of finding the price of the American put option is often described in terms of the free boundary problem (3.3) without the two inequalities above. Nevertheless, the inequalities above are necessary for the variational formulation (2.6). Indeed, it is the reason why $b(0)=b_{0}$. To see this, recall that

$$
\mathcal{L} p_{0}(x)=\delta(x)+k\left[e^{x-b_{0}}-1\right] H(-x)
$$

where $H$ is the Heaviside function, $H(z)=1$ for $z>0$ and $H(z)=0$ for $z<0$, and $\delta(x)=H^{\prime}(x)$ is the Delta function. It is critical here that $\mathcal{L} p_{0}$ changes sign only once:

$$
\mathcal{L} p_{0} \geqslant 0 \quad \text { in }\left[b_{0}, \infty\right), \quad \mathcal{L} p_{0}<0 \quad \text { in }\left(-\infty, b_{0}\right) ; \quad b_{0}:=\min \{0, \ln (k / \ell)\}
$$

This property implies that the free boundary in (2.6) is well-defined. Indeed, for each $s>0$,

$$
p(x, s)>p_{0}(x) \quad \forall x>b(s), \quad p(x, s)=p_{0}(x) \quad \forall x \leqslant b(s) .
$$

Furthermore, the continuity and the first two sets of inequalities in (3.3) imply that $b(0)=b_{0}$.

\section{Proof of Theorem 3.1}

\section{Idea of the Proof}

Chen and Chadam [15] showed that the problem (2.6) has a unique solution for the case $l=0$ (i.e., $D=0$ ) by using a penalty method [19]. Here we consider the general case by using the technique introduced in [17].

In [17], the analysis is carried out in terms of the function $q:=p_{s}$. Formally one can derive that $(q, b)$ is a solution of the following free boundary problem:

$$
\begin{cases}q_{s}(x, s)=\mathcal{L} q(x, s) & \forall x>b(s), s>0, \\ q(x, s)=0 & \forall x \leqslant b(s), s>0, \\ q(x, 0)=\max \left\{\mathcal{L} p_{0}(x), 0\right\} & \forall x \in \mathbb{R}, \quad s=0, \\ \Pi(b(s))=\int_{0}^{s} q_{x}(b(t), t) d t & \forall s \geqslant 0,\end{cases}
$$


where

$$
\Pi(z)=\int_{\infty}^{z} \min \left\{\mathcal{L} p_{0}(x), 0\right\} d x= \begin{cases}0 & \text { if } z \geqslant b_{0} \\ \int_{b_{0}}^{z}\left(\ell e^{x}-k\right) d x & \text { if } z<b_{0}\end{cases}
$$

Here the last equation in (3.4) is a weak formulation of the free boundary condition

$$
b(0)=b_{0}, \quad \dot{b}(s)\left[\ell e^{b(s)}-k\right]=q_{x}(b(s), s), \quad b(s) \leqslant b_{0} \quad \forall s>0 .
$$

The problem (3.4) is a Stefan type free boundary problem which has been well-studied (see, for example, $[21,26]$ ). The existence of a smooth classical solution would be standard if the free boundary condition were not degenerate; i.e., the coefficient $\left(\ell e^{b}-k\right)$ of $\dot{b}$ at $s=0$ in (3.5) is not zero. We shall treat this degeneracy by using the initial value $b(0)=b_{0}-\epsilon$ for positive $\epsilon$ and then sending $\epsilon \searrow 0$. Thus, we shall study the approximation problem for $\left(q^{\epsilon}, b^{\epsilon}\right)$ :

$$
\begin{cases}q_{s}^{\epsilon}(x, s)=\mathcal{L} q^{\epsilon}(x, s) & \forall x>b^{\epsilon}(s), s>0, \\ q^{\epsilon}(x, s)=0 & \forall x \leqslant b^{\epsilon}(s), s>0, \\ q^{\epsilon}(x, 0)=\max \left\{\mathcal{L} p_{0}(x), 0\right\} & \forall x \in \mathbb{R}, \quad s=0, \\ \tilde{\Pi}\left(b^{\epsilon}(s)\right)=\tilde{\Pi}\left(b_{0}-\epsilon\right)+\int_{0}^{s} q_{x}(b(t), t) d t & \forall s \geqslant 0 .\end{cases}
$$

where $\tilde{\Pi}=\Pi$ if $\alpha=(k-\ell-1) \leqslant 0$ and

$$
\tilde{\Pi}(z)= \begin{cases}\Pi(z) & \text { if } z \geqslant b^{*} \\ \Pi\left(b^{*}\right)+\int_{b_{0}}^{z}\left(\ell e^{x}-k\right) e^{\alpha\left(b^{*}-x\right) / 2} d x & \text { if } z<b^{*}\end{cases}
$$

if $\alpha>0$. Here $b^{*}=\ln (\lambda / 1+\lambda)$ and $\lambda:=\left(\alpha+\sqrt{\alpha^{2}+4 k}\right) / 2$. In the end, we shall show that $b \geqslant b^{*}$ so that $\Pi(b)=\tilde{\Pi}(b)$.

\section{Existence of the approximation problem}


Lemma 3.1. For each $\epsilon>0$, the approximation problem (3.6) admits a solution $\left(q^{\epsilon}, b^{\epsilon}\right)$ satisfying $q^{\epsilon} \in \cap_{0<\beta<1}^{2 \beta, \beta}\left(\mathbb{R} \times(0, \infty)\right.$ and $b^{\epsilon} \in C^{1}([0, \infty))$. In addition, for any positive constant $\eta \geqslant-b_{0}$

$$
0 \leqslant e^{\alpha b^{\epsilon}(s) / 2}\left[\ell e^{b^{\epsilon}(s)}-k\right] \dot{b}^{\epsilon}(s) \leqslant M(\eta) e^{-\left[k+\alpha^{2} / 4\right] s} \quad \text { if } \quad b^{\epsilon}(s) \leqslant-\eta
$$

where $M(\cdot)$ is defined by

$$
M(\eta):=\max \left\{M_{0}, \sup _{t>0} \frac{\tilde{q}_{0}(-\eta / 2, t)}{\eta / 2}\right\}
$$

with

$$
\begin{aligned}
\tilde{q}_{0}(x, s) & =\frac{e^{-x^{2} /(4 s)}}{\sqrt{4 \pi s}}+\int_{b_{0}}^{0} \frac{e^{-(x-y)^{2} /(4 s)}}{\sqrt{4 \pi s}} e^{\alpha y / 2}\left(\ell e^{y}-k\right) d y \\
M_{0} & :=\max _{x \in\left[b_{0}, 0\right]}\left|\frac{d\left[e^{\alpha x / 2}\left(\ell e^{x}-k\right)\right]}{d x}\right| .
\end{aligned}
$$

Proof. The one space dimensional Stefan problem can be solved as follows. We establish the existence of a solution in a time interval $[0, h]$ for an arbitrary large $h$. For this we define a function space

$$
\mathbf{B}=\left\{b \in C^{1}([0, h]) \mid b(0)=b_{0}-\epsilon ; 0 \leqslant e^{\alpha b / 2}\left[\ell e^{b}-k\right] \dot{b} \leqslant M\left(\epsilon-b_{0}\right) \text { in }[0, h]\right\}
$$

where $M(\cdot)$ is defined in (3.8). Clearly, $\mathbf{B}$ is a closed subset of $C^{1}([0, h])$.

For each $b \in \mathbf{B}$, we let $q$ be the solution of the following initial boundary value problem

$$
\begin{cases}q_{s}=\mathcal{L} q & \text { in } Q_{b}:=\{(x, s) \mid s>0, x>b(s)\}, \\ q(b(s), s)=0 & \forall s>0 \\ q(x, 0)=\max \left\{\mathcal{L} p_{0}(x), 0\right\} & \forall x \geqslant b_{0}-\epsilon .\end{cases}
$$

This problem admits a unique classical solution $q$ [20]. As the lateral boundary of $Q_{b}$ is $C^{1}$, we see that $q \in \cap_{0<\beta<1} C^{2 \beta, \beta}\left(\overline{Q_{b}} \backslash\left(\left[b_{0}, 0\right] \times\{0\}\right)\right)$ which implies $q_{x}(b(\cdot), \cdot) \in \cap_{0<\beta<1 / 2} C^{\beta}([0, h])$.

For each $b \in \mathbf{B}$, using the solution $q$, we define $\tilde{b}=\mathbf{T}[b]$ by solving the ode

$$
e^{\alpha \hat{b}(s) / 2}\left[\ell e^{\tilde{b}(s)}-k\right] \frac{d \tilde{b}(s)}{d s}=q_{x}(b(s), s) e^{\alpha b(s) / 2}, \quad \forall s \in(0, h] \quad \tilde{b}(0)=b_{0}-\epsilon,
$$

where $\hat{b}(s)=\tilde{b}(s)$ if $\alpha \leqslant 0$ and $\hat{b}(s)=\max \left\{b^{*}, \tilde{b}(s)\right\}$ if $\alpha>0$. 
Here the special modification of replacing $e^{\alpha \tilde{b}}$ by $e^{\alpha \hat{b}}$ (when $\alpha>0$ ) ensures that ODE problem (3.10) has a global solution since such choice of $\hat{b}(s)$ can prevent $\tilde{b}(s)$ from being unbounded from below before $h$. Since $q>0$ in $Q_{b}$, we have $q_{x}(b(s), s) \geqslant 0$. Hence, $\tilde{b}$ is well-defined and $\tilde{b}(s) \leqslant b_{0}-\epsilon$ for all $s \in[0, h]$.

A fixed point of $\mathbf{T}$ is a solution of (3.6). To show that $\mathbf{T}$ has a fixed point in $\mathbf{B}$, we first show that $\mathbf{T}$ maps $\mathbf{B}$ to itself. For this, we need an upper bound for $\tilde{b}$. Let $q_{0}$ be the solution of

$$
q_{0 s}=\mathcal{L} q_{0} \quad \text { on } \mathbb{R} \times(0, \infty), \quad q_{0}(\cdot, 0)=\max \left\{\mathcal{L} p_{0}, 0\right\}
$$

Then by the maximum principle $q_{0}>0$ on $\mathbb{R} \times(0, \infty)$, and by comparison $q \leqslant q_{0}$ on $\mathbb{R} \times[0, \infty)$.

Define $\tilde{q}_{0}:=q_{0}(x, s) e^{\alpha x / 2+\left(k+\alpha^{2} / 4\right) s}$. Then $\tilde{q}_{0}$ satisfies $\tilde{q}_{0 s}=\tilde{q}_{0 x x}$ on $\mathbb{R} \times(0, \infty)$ with initial data $\delta(x)+e^{\alpha x / 2}\left(\ell e^{x}-k\right) H(-x) H\left(x-b_{0}\right)$. Hence, $\tilde{q}_{0}$ is given by (3.9). Note that $\left\|\tilde{q}_{0}(x, \cdot)\right\|_{L^{\infty}([0, \infty))}$ is finite for every $x \neq 0$. Let $\eta$ be any positive constant such that $\eta \geqslant-b_{0}$. Define $M(\eta)$ as in (3.8) and for each $b \in \mathbf{B}$, define

$$
\begin{aligned}
b^{\eta}(s) & :=\min \{-\eta, b(s)\}, \\
q_{1}(x, s) & :=M(\eta)\left(x-b^{\eta}(s)\right) e^{-\alpha x / 2-\left(k+\alpha^{2} / 4\right) s} .
\end{aligned}
$$

Since $\dot{b} \leqslant 0, b^{\eta}$ is a continuous decreasing function. Notice that

$$
q_{1 s}-\mathcal{L} q_{1}=-M(\eta)\left[x-b^{\eta}(s)\right] \dot{b}^{\eta}(s) e^{-\alpha x / 2-\left(k+\alpha^{2} / 4\right) s} \geqslant 0 \quad \forall x \geqslant b^{\eta}(s), s \geqslant 0 .
$$

Now we compare $q_{1}$ with $q$ on $\bar{Q}$ where $Q=\{(x, s) \mid b(s)<x<-\eta / 2, s>0\}$. Since $q \leqslant q_{0}$ on $\mathbb{R} \times(0, \infty)$ and $q=0$ when $x \leqslant b(s)$, our choice of $M(\eta)$ implies that $q_{1} \geqslant q$ on the parabolic boundary of $Q$, so $q<q_{1}$ in $Q$.

Suppose $b(s) \leqslant-\eta$. Then $q(b(s), s)=q_{1}(b(s), s)=0$ and $q(x, s)<q_{1}(x, s)$ for $x \in$ $(b(s),-\eta / 2]$. Hence,

$$
0 \leqslant q_{x}(b(s), s) \leqslant q_{1 x}(b(s), s)=M(\eta) e^{-\alpha b(s) / 2-\left(k+\alpha^{2} / 4\right) s} \quad \text { if } b(s) \leqslant-\eta .
$$


It then follows from (3.10) that

$$
0 \leqslant e^{\alpha \hat{b} / 2}\left[\ell e^{\tilde{b}}-k\right] \dot{\tilde{b}} \leqslant M(\eta) e^{-\left(k+\alpha^{2} / 4\right) s} .
$$

In particular, letting $\eta=\epsilon-b_{0}$ in the above inequality, one obtains

$$
0 \leqslant e^{\alpha \hat{b} / 2}\left[\ell e^{\tilde{b}}-k\right] \dot{\tilde{b}} \leqslant M\left(\epsilon-b_{0}\right) e^{-\alpha b(s) / 2-\left(k+\alpha^{2} / 4\right) s}
$$

Finally, since $e^{\alpha \tilde{b} / 2} \leqslant e^{\alpha \hat{b} / 2}$, we have

$$
0 \leqslant e^{\alpha \tilde{b} / 2}\left[\ell e^{\tilde{b}}-k\right] \dot{\tilde{b}} \leqslant M\left(\epsilon-b_{0}\right) e^{-\alpha b(s) / 2-\left(k+\alpha^{2} / 4\right) s}
$$

Thus, $\mathbf{T}$ maps $\mathbf{B}$ to itself. In addition $\{\mathbf{T}[b] \mid b \in \mathbf{B}\}$ is a bounded set in $C^{1+1 / 4}([0, h])$ which is a compact subset of $C^{1}([0, h])$. Hence, by the Schauder's fixed point theorem, $\mathbf{T}$ admits a fixed point, $b^{\epsilon}$, in $\mathbf{B}$. Moreover since $M\left(\epsilon-b_{0}\right)$ does not depend on $h$, one can let $h \rightarrow \infty$ to obtain $b^{\epsilon} \in C^{1}([0, \infty))$. Extend the corresponding $q$ by zero for $x<b^{\epsilon}$ and denote it by $q^{\epsilon}$. We see that $\left(q^{\epsilon}, b^{\epsilon}\right)$ is a classical solution of (3.6).

\section{Limits of the approximation sequence}

Lemma 3.2. Let $\left(q^{\epsilon}, b^{\epsilon}\right)$ be the solution of the problem (3.6). Then the sequence $\left(q^{\epsilon}, b^{\epsilon}\right)$ is monotonic, i.e., if $0<\epsilon_{1}<\epsilon_{2}$, then $b^{\epsilon_{2}}<b^{\epsilon_{1}}$ and $q^{\epsilon_{1}}<q^{\epsilon_{2}}$. Consequently, there exist $q$ and b such that

$$
q=\lim _{\epsilon \searrow 0} q^{\epsilon}, \quad b=\lim _{\epsilon \searrow 0} b^{\epsilon}
$$


Proof. Since $b^{\epsilon}(0)=b_{0}-\epsilon$, clearly, $b^{\epsilon_{2}}(0)<b^{\epsilon_{1}}(0)$ if $0<\epsilon_{1}<\epsilon_{2}$. We claim that $b^{\epsilon_{2}}<b^{\epsilon_{1}}$ on $[0, \infty)$. Suppose this is not true. Then $t^{*}:=\sup \left\{t>0 \mid b^{\epsilon_{2}}<b^{\epsilon_{1}}\right.$ in $\left.[0, t]\right\}$ is finite and we have $b^{\epsilon_{2}}<b^{\epsilon_{1}}$ in $\left[0, t^{*}\right)$ and $b^{\epsilon_{2}}\left(t^{*}\right)=b^{\epsilon_{1}}\left(t^{*}\right)$.

Now by comparison on $D=\left\{(x, t) \mid x \geqslant b^{\epsilon_{1}}(s), s \in\left[0, t^{*}\right]\right\}$ we see that $q^{\epsilon_{2}} \geqslant q^{\epsilon_{1}}$ on $D$. Since $b^{\epsilon_{1}}$ is $C^{1}$, we obtain from Hopf's lemma [22, see Theorem 2.8 in p78] that $q_{x}^{\epsilon_{2}}\left(b^{\epsilon_{1}}\left(t^{*}\right), t^{*}\right)>q_{x}^{\epsilon_{1}}\left(b^{\epsilon_{1}}\left(t^{*}\right), t^{*}\right)$. Consequently, since $b^{\epsilon_{1}}\left(t^{*}\right)=b^{\epsilon_{2}}\left(t^{*}\right)$ and then $\hat{b}^{\epsilon_{1}}\left(t^{*}\right)=$ $\hat{b}^{\epsilon_{2}}\left(t^{*}\right)$, we find from the boundary condition that

$$
-\dot{b}^{\epsilon_{2}}\left(t^{*}\right)=\frac{q_{x}^{\epsilon_{2}}\left(b^{\epsilon_{1}}\left(t^{*}\right), t^{*}\right) e^{\alpha\left(b^{\epsilon_{2}}-\hat{b}^{\epsilon_{2}}\right) / 2}}{k-e^{b_{1}\left(t^{*}\right)}}>\frac{q_{x}^{\epsilon_{1}}\left(b^{\epsilon_{1}}\left(t^{*}\right), t^{*}\right) e^{\alpha\left(b^{\epsilon_{1}}-\hat{b}^{\epsilon_{1}}\right) / 2}}{k-e^{\epsilon_{1}}\left(t^{*}\right)}=-\dot{b}^{\epsilon_{1}}\left(t^{*}\right) .
$$

That is $\left.\left(b^{\epsilon_{2}}-b^{\epsilon_{1}}\right)^{\prime}\right|_{s=t^{*}}<0$. But this implies $b^{\epsilon_{2}}(s)-b^{\epsilon_{1}}(s)>0$ when $0<t^{*}-s \ll 1$, contradicting the definition of $t^{*}$. Hence, we must have $b^{\epsilon_{2}}<b^{\epsilon_{1}}$ on $[0, \infty)$. Consequently, by comparison, we have $q^{\epsilon_{1}}<q^{\epsilon_{2}}$ in $Q_{b^{\varepsilon_{1}}}$. Therefore, the sequence $\left(q^{\epsilon}, b^{\epsilon}\right)$ is convergent.

\section{Regularity of $b$}

Lemma 3.3. Let $(q, b)$ be defined as in lemma 3.2. Then $(q, b)$ is a solution of problem (3.4) and $b \in C^{\infty}((0, \infty)) \cap C([0, \infty))$.

Proof. Define $\hat{t}=\sup \left\{t>0 \mid b>b^{*}\right.$ in $\left.[0, t]\right\}$. Later, in the next section we shall provide an independent proof that $\hat{t}=\infty$. So, in this section, we assume that $\hat{t}=\infty$. As the limit of a sequence of decreasing functions, $b$ is also decreasing.

We claim that $b(s)<b_{0}$ for every $s>0$. Indeed, if this is not true, then we have $b(s)=b_{0}$ for all $s \in[0, \delta]$ for some $\delta>0$. Note that for the $\epsilon$ problem, integrating $q_{s}^{\epsilon}=\mathcal{L} q^{\epsilon}$ over $Q_{b^{\epsilon}}$, we have the following identity, for $t_{2}>t_{1} \geqslant 0$,

$$
\begin{array}{r}
\int_{\mathbb{R}} q^{\epsilon}\left(x, t_{2}\right) d x-\int_{\mathbb{R}} q^{\epsilon}\left(x, t_{1}\right) d x+k \int_{t_{1}}^{t_{2}} \int_{\mathbb{R}} q^{\epsilon}(x, t) d x d t \\
=-\int_{t_{1}}^{t_{2}} q_{x}^{\epsilon}\left(b^{\epsilon}(t), t\right) d t=\tilde{\Pi}\left(b^{\epsilon}\left(t_{1}\right)\right)-\tilde{\Pi}\left(b^{\epsilon}\left(t_{2}\right) .\right.
\end{array}
$$

Sending $\epsilon \searrow 0$ and using Lebesgue's dominated theorem we obtain

$$
\int_{\mathbb{R}} q\left(x, t_{2}\right)-\int_{\mathbb{R}} q\left(x, t_{1}\right) d x+k \int_{t_{1}}^{t_{2}} \int_{\mathbb{R}} q(x, t) d x d t=\tilde{\Pi}\left(b\left(t_{1}\right)-\tilde{\Pi}\left(b\left(t_{2}\right)\right) \quad \forall t_{2}>t_{1} \geqslant 0 .\right.
$$


Now if $b \equiv b_{0}$ on $[0, \delta]$, we can integrate $q_{s}=\mathcal{L} q$ over $\left(0, t^{\epsilon_{0}}\right) \times(\delta / 2, \delta)$ to derive

$$
\int_{\delta / 2}^{\delta} q_{x}\left(b_{0}, t\right) d t=\tilde{\Pi}(b(\delta)-\tilde{\Pi}(b(\delta / 2))=0,
$$

which is impossible since Hopf's maximum principle implies that $q_{x}\left(b_{0}, s\right)>0$ for each $s \in(0, \delta)$. In conclusion, $b(s)<b_{0}$ for every $s>0$.

Now let $\eta \geqslant-b_{0}$ be any small positive constant and $M(\eta)$ be defined as in (3.8). For each $\epsilon>0$, let $\left(q^{\epsilon}, b^{\epsilon}\right)$ be the solution of (3.6). By (3.12), one has

$$
0 \leqslant q_{x}^{\epsilon}(b(s), s) \leqslant M(\eta) e^{-\alpha b^{\epsilon}(s) / 2-\left(k+\alpha^{2} / 4\right) s} \quad \text { if } \quad b^{\epsilon}(s) \leqslant-\eta
$$

It then follows from the last equation in (3.6) that

$$
0 \leqslant e^{\alpha \hat{b}^{\epsilon}(s) / 2}\left[\ell e^{b^{\epsilon}(s)}-k\right] \dot{b}^{\epsilon}(s) \leqslant M(\eta) e^{-\left[k+\alpha^{2} / 4\right] s} \quad \text { if } \quad b^{\epsilon}(s) \leqslant-\eta .
$$

Sending $\epsilon \searrow 0$, we then obtain

$$
0 \leqslant e^{\alpha \hat{b}(s) / 2}\left[\ell e^{b(s)}-k\right] \dot{b}(s) \leqslant M(\eta) e^{-\left[k+\alpha^{2} / 4\right] s} \quad \text { if } \quad b(s) \leqslant-\eta
$$

This implies that $b$ is Lipschitz continuous on $[\delta, \infty)$, where $\delta:=\inf \{s>0 \mid b(s)<-\eta\}$.

Now we can use $\left(q^{\varepsilon}, b^{\epsilon}\right) \rightarrow(q, b)$ to conclude that $q(b(s), s)=0$ and $q_{x}(b(s), s)=\dot{b}\left[\ell e^{b(s)}-\right.$ $k$ ] for all $s \in(\delta, \infty)$. Next we claim that that $b \in C^{\infty}((\delta, \infty))$ by the following bootstrap argument. Notice that $b \in C^{\beta / 2}((0, \infty))$ for some $\beta>1$ which is not an integer. Then by standard local regularity theory, e.g. potential theory [20, see chapter 5], the solution of $q_{s}=\mathcal{L} q$ in $Q_{b}$ with zero boundary value on $x=b(s)$ has the regularity $q \in C^{\beta, \beta / 2}(D)$ and $q_{x} \in C^{\beta-1,(\beta-1) / 2}(D)$ where $D:=\{(x, s) \mid x \geqslant b(s), s>\delta\}$. Consequently, $q_{x}(b(\cdot), \cdot) \in$ $C^{(\beta-1) / 2}((\delta, \infty))$. Since $\ell e^{b(s)}-k<0$ for all $s>0$, the last equation in (3.4) can be differentiated to give $\dot{b}\left[\ell e^{b}-k\right]=q_{x}(b, s)$, from which we conclude that $b \in C^{(\beta+1) / 2}((\delta, \infty))$. Thus, by induction, $b \in C^{\infty}((\delta, \infty))$.

Sending $\eta \searrow-b_{0}$, we must have $\delta \rightarrow 0$ since $b(s)<b_{0}$ for each $s>0$. Thus, $b \in$ $C^{\infty}((0, \infty))$ and $(q, s)$ is a solution of $(3.4)$.

Finally, we show that $b$ is continuous at $s=0$. Let $\delta^{\epsilon}:=\inf \left\{s>0 \mid b^{\epsilon}(s)>b_{0}-2 \epsilon\right\}$. Then $\delta^{\epsilon} \in(0, \infty]$ and $b(s) \geqslant b^{\epsilon}>b_{0}-2 \epsilon$ for all $s \in\left(0, \delta^{\epsilon}\right)$. Hence, $\underline{\lim }_{s \searrow 0} b(s) \geqslant b_{0}-2 \epsilon$. Sending 
$\epsilon \searrow 0$ we find $\underline{\lim }_{s \searrow 0} b(s) \geqslant b_{0}$. As $b(s)<b_{0}$ for $s>0$, we conclude that $\lim _{s \rightarrow 0} b(s)=b_{0}$, so $b \in C([0, \infty)$.

\section{Recovering $p$ from $q$}

Lemma 3.4. Let $(q, b)$ be the classical solution of (3.4) as in lemma 3.3. Define

$$
p(x, s):=p_{0}(x)+\int_{0}^{s} q(x, t) d t \quad \forall x \in \mathbb{R}, s \geqslant 0,
$$

$(p, b)$ solves the problem (3.3), p solves problem (2.6), and b satisfies (2.7).

Proof. Since $q(x, t)=0$ for $x \leqslant b(t)$ and since $\dot{b}<0$, we see that $q=0$ on $(-\infty, b(s)] \times[0, s]$ for each $\hat{t}>s>0$. Hence, $p(x, s) \equiv p_{0}(x)$ when $x \leqslant b(s)$ and $p_{x}(b(s), s)=p_{0 x}(b(s))$ for each $\hat{t}>s>0$. Also, $p_{s}=q$ on $\mathbb{R} \times(0, \infty)$. In addition, when $x \geqslant b_{0}$,

$$
\mathcal{L} p(x, s)=\mathcal{L} p_{0}(x)+\int_{0}^{s} \mathcal{L} q(x, t) d t=\mathcal{L} p_{0}+\int_{0}^{s} q_{t}(x, t) d t=q(x, s)=p_{s}
$$

since $q(\cdot, 0)=\max \left\{\mathcal{L} p_{0}, 0\right\}=\mathcal{L} p_{0}$ when $x \geqslant b_{0}$.

When $x \in\left(b(s), b_{0}\right)$, write $s=\hat{s}(x)$ the inverse of $x=b(s)$. Then

$$
p(x, t)=p_{0}(x)+\int_{\hat{s}(x)}^{s} q(x, t) d t .
$$

Consequently,

$$
\begin{aligned}
\mathcal{L} p(x, s) & =\mathcal{L} p_{0}(x)-\hat{s}^{\prime}(x) q_{x}(x, \hat{s}(x))+\int_{\hat{s}(x)}^{s} \mathcal{L} q(x, t) d t \\
& =\mathcal{L} p_{0}(x)-\frac{1}{\dot{b}(\hat{s}(x))} q_{x}(x, \hat{s}(x))+q(x, s)=q(x, s)=p_{s}
\end{aligned}
$$

Thus, $(p, b)$ is a solution of the variational inequality $(2.6)$ in $\mathbb{R} \times[0, \hat{t})$.

Now we claim $\hat{t}=\infty$. Let $\left(p^{*}(\cdot), b^{*}\right) \in C^{1}(\mathbb{R}) \times \mathbb{R}$ be the well-known Merton solution of

$$
p^{*}=p_{0} \quad \text { on }\left(-\infty, b^{*}\right], \quad p^{* \prime \prime}+\alpha p^{* \prime}-k p^{*}=0 \quad \text { in }\left(b^{*}, \infty\right), \quad p^{*}(\infty)=0 .
$$

The solution $p^{*}$ is given by

$$
p^{*}(x):=\max \left\{1-e^{x}, \frac{e^{-\lambda\left(x-b^{*}\right)}}{1+\lambda}\right\}
$$


and $b^{*}$ is the same as the defined in (3.6). Following the proof of Theorem 2.3 in [15], one can show that $p \leqslant p^{*}, b^{*} \leqslant b$ by the comparison theorem. It then follows that $\hat{t}=\infty$.

Finally, $p_{0}=q>0$ in $Q_{b}$, so $p>p_{0}$ in $Q_{b}$. Also when $x<b(s), p=p_{0}$ and $\mathcal{L} p-p_{s}=$ $\mathcal{L} p_{0}=k\left[e^{x-b_{0}}-1\right]<0$. Thus, $p$ is a solution of (3.3) and also a solution of the obstacle problem (2.6).

Since the solution of the variational inequality (2.6) is unique (see [20] chapter 1 and [15]), the assertion of Theorem 3.1 thus follows.

Remark 3.1. Let $p^{\epsilon}:=p_{0}+\int_{0}^{t} q^{\epsilon}(x, t) d t$. Then $\left(p^{\epsilon}, b^{\epsilon}\right)$ does not solve the original problem; one finds that $p_{s}^{\epsilon}-\mathcal{L} p^{\epsilon}=-\mathcal{L} p_{0}(x)$ in $\left(b_{0}-\epsilon, b_{0}\right) \times(0, \infty)$. Indeed, as $\epsilon \searrow 0, p^{\epsilon} \searrow p$ and $b^{\epsilon} \nearrow b$. 


\subsection{INTEGRAL EQUATIONS}

In addition to the variational formulation of the American put problem (2.6)-(2.7), it is useful to use an approach based on integral equation for the free boundary. In this chapter we develop several integral equations which are useful for our analysis and numerical simulation in the later chapters.

Let $p(x, s)$ be defined in $\mathbb{R} \times(0, \infty)$ from problem $(2.6)-(2.7)$, then $\phi(x, s):=p(x, s)-p_{0}(x)$ satisfies

$$
\begin{aligned}
& \phi_{s}-\mathcal{L} \phi(x, s)=H(x-b(s)) \mathcal{L} p_{0}(x) \text { on } \mathbb{R} \times(0, \infty), \\
& \phi(\cdot, 0)=0 \text { on } \mathbb{R} \times\{0\} .
\end{aligned}
$$

Where $H$ is the heaviside function. Using Green's formula, one can write the solution of (4.1) as

$$
\phi(x, s)=\int_{0}^{s} \int_{b(s-t)}^{\infty} \Gamma(x-y, t) \mathcal{L} p_{0}(y) d y d t, \quad \forall(x, s) \in \mathbb{R} \times[0, \infty),
$$

where $\Gamma$ is the fundamental solution given by

$$
\Gamma(x, s):=K(x+\alpha s, s) e^{-k s}, \quad K(z, t):=\frac{1}{\sqrt{4 \pi t}} e^{-z^{2} / 4 t} .
$$

Noting that

$$
\mathcal{L} p_{0}(x)=\delta(x)+\left(l e^{x}-k\right) H(-x),
$$

one can rewrite (4.2) as

$$
\phi(x, s)=\int_{0}^{s} \Gamma(x, t) d t+\int_{0}^{s} \int_{b(s-t)}^{0}\left[\ell e^{y}-k\right] \Gamma(x-y, t) d y d t,
$$


Differentiating the above equation with respect to the variables $x, s$ yields, for every $(x, s) \in$ $\mathbb{R} \times(0, \infty)$,

$$
\phi_{x}(x, s)=\int_{0}^{s} \Gamma_{x}(x, t) d t+\int_{0}^{s} \int_{b(s-t)}^{0}\left[\ell e^{y}-k\right] \Gamma_{x}(x-y, t) d y d t
$$

and

$$
\begin{aligned}
\phi_{s}(x, s)= & \Gamma(x, s)+\int_{b(0)}^{0}\left[\ell e^{y}-k\right] \Gamma(x-y, s) d y \\
& -\int_{0}^{s} \dot{b}(s-t)\left[\ell e^{b(s-t)}-k\right] \Gamma(x-b(s-t), t) d t \\
= & \Gamma(x, s)+\int_{b_{0}}^{0}\left[\ell e^{y}-k\right] \Gamma(x-y, s) d y \\
& -\int_{0}^{s} \dot{b}(t)\left[\ell e^{b(t)}-k\right] \Gamma(x-b(t), s-t) d t
\end{aligned}
$$

Moreover, for every $s>0$ and $x \neq b(s)$,

$$
\begin{aligned}
\phi_{s x}(x, s)=\Gamma_{x}(x, s) & +\int_{b_{0}}^{0}\left[\ell e^{y}-k\right] \Gamma_{x}(x-y, s) d y \\
& -\int_{0}^{s} \dot{b}(t)\left[\ell e^{b(t)}-k\right] \Gamma_{x}(x-b(t), s-t) d t .
\end{aligned}
$$

Evaluating these expressions at $x=b(s)$ one then obtains the following theorem.

Theorem 4.1. Let $(p, b)$ be the solution of the variational inequality (2.6)-(2.7). Then $b$ satisfies the following integral identities:

$$
\begin{aligned}
& 0=\int_{0}^{s} \Gamma(b(s), t) d t+\int_{0}^{s} \int_{b(s-t)}^{0}\left[\ell e^{y}-k\right] \Gamma(b(s)-y, t) d y d t, \\
& 0=\int_{0}^{s} \Gamma_{x}(b(s), t) d t+\int_{0}^{s} \int_{b(s-t)}^{0}\left[\ell e^{y}-k\right] \Gamma_{x}(b(s)-y, t) d y d t \\
& 0=\Gamma(b(s), s)+\int_{b_{0}}^{0}\left[\ell e^{y}-k\right] \Gamma(b(s)-y, s) d y \\
& -\int_{0}^{s} \dot{b}(t)\left[\ell e^{b(t)}-k\right] \Gamma(b(s)-b(t), s-t) d t \\
& \dot{b}(s)\left[\ell e^{b(s)}-k\right]=2 \Gamma_{x}(b(s), s)+2 \int_{b_{0}}^{0}\left[\ell e^{y}-k\right] \Gamma_{x}(b(s)-y, s) d y \\
& -2 \int_{0}^{t} \dot{b}(t)\left[\ell e^{b(t)}-k\right] \Gamma_{x}(b(s)-b(t), s-t) d t .
\end{aligned}
$$


Also, for any $\theta \in \mathbb{R}$,

$$
\begin{aligned}
\dot{b}(s)\left[\ell e^{b(s)}-k\right]= & \left(\theta-\frac{b(s)}{s}\right) \Gamma(b(s), s) \\
& +\int_{b_{0}}^{0}\left[\ell e^{y}-k\right]\left(\theta-\frac{b(s)-y}{s}\right) \Gamma(b(s)-y, s) d y \\
& -\int_{0}^{s} \dot{b}(t)\left[\ell e^{b(t)}-k\right]\left(\theta-\frac{b(s)-b(t)}{s-t}\right) \Gamma(b(s)-b(t), s-t) d t .
\end{aligned}
$$

Proof. Let $(p, b)$ be the solution of the problem (2.6), then the identities (4.3)-(4.5) must hold. From the boundary values (3.1), one obtains

$$
\phi(b(s), s)=\phi_{x}(b(s), s)=\phi_{s}(b(s), s)=0
$$

which imply (4.8)-(4.10) directly. Since from (3.1e) $p_{s x}(b(s)+, s)=\dot{b}(s)\left(\ell e^{b(s)}-k\right)$,

$$
\phi_{s x}(b(s)+, s)=\dot{b}(s)\left(\ell e^{b(s)}-k\right) .
$$

Applying this in (4.5) and taking into account the jump discontinuity of $\Gamma$, one obtains

$$
\begin{aligned}
\dot{b}(s)\left(\ell e^{b(s)}-k\right)=\lim _{x \rightarrow b(s)} & {\left[\Gamma_{x}(x, s)+\int_{b_{0}}^{0}\left[\ell e^{y}-k\right] \Gamma_{x}(x-y, s) d y\right.} \\
& \left.-\int_{0}^{s} \dot{b}(t)\left[\ell e^{b(t)}-k\right] \Gamma_{x}(x-b(t), s-t) d t\right] \\
=\Gamma_{x}(b(s), s)+\int_{b_{0}}^{0}\left[\ell e^{y}-k\right] \Gamma_{x}(b(s)-y, s) d y & \\
& -\int_{0}^{t} \dot{b}(t)\left[\ell e^{b(t)}-k\right] \Gamma_{x}(b(s)-b(t), s-t) d t+\frac{\dot{b}(s)\left(\ell e^{b(s)}-k\right)}{2},
\end{aligned}
$$

which leads to (4.10).

Using the fact

$$
\Gamma_{x}(x, s)=\frac{1}{2}\left(\frac{x}{s}+\alpha\right) \Gamma(x, s),
$$

the above identity becomes

$$
\begin{aligned}
\dot{b}(s)\left(\ell e^{b(s)}-k\right)= & \left(\frac{b(s)}{s}+\alpha\right) \Gamma(b(s), s) \\
& +\int_{b_{0}}^{0}\left[\ell e^{y}-k\right]\left(\frac{b(s)-y}{s}+\alpha\right) \Gamma(b(s)-y, s) d y \\
& -\int_{0}^{t} \dot{b}(t)\left[\ell e^{b(t)}-k\right]\left(\frac{b(s)-b(t)}{s-t}+\alpha\right) \Gamma(b(s)-b(t), s-t) d t .
\end{aligned}
$$

For any $\theta \in \mathbb{R}, s \in \mathbb{R}$, multiplying (4.10) by $\theta+\alpha$ and subtracting it from (4.12), one has the integro-differential equation (4.11) 
We shall use (4.11) with particular choices of $\theta$ for our analysis and numerical simulation. 


\subsection{NUMERICAL SIMULATION}

In this chapter, we provide an accurate and fast numerical scheme to solve the integrodifferential equation of the free boundary (see equation (4.11))

$$
\begin{aligned}
\dot{b}(s)\left[\ell e^{b(s)}-k\right]= & \left(\theta-\frac{b(s)}{s}\right) \Gamma(b(s), s) \\
& +\int_{b_{0}}^{0}\left[\ell e^{y}-k\right]\left(\theta-\frac{b(s)-y}{s}\right) \Gamma(b(s)-y, s) d y \\
& -\int_{0}^{s} \dot{b}(t)\left[\ell e^{b(t)}-k\right]\left(\theta-\frac{b(s)-b(t)}{s-t}\right) \Gamma(b(s)-b(t), s-t) d t .
\end{aligned}
$$

with $\theta$ chosen to remove the singularity in the last integral.

\subsection{NUMERICAL SCHEME}

There is a singularity in the last integral of equation (5.1) when $s$ is close to zero. From the analysis to be provided in chapter 7 , the asymptotic expansion gives, for $l>k$,

$$
b(s) \sim b_{0}-A_{0} \sqrt{s}, \quad \dot{b}(s) \sim-\frac{A_{0}}{2 \sqrt{s}}, \quad \text { as } s \searrow 0
$$

where $A_{0}=0.90344659 \ldots$ Hence when $0<s \ll 1$

$$
\lim _{t \rightarrow s-} \frac{b(s)-b(t)}{s-t}=\dot{b}(s) \sim-\frac{A_{0}}{2 \sqrt{s}} \sim \frac{b(s)-b_{0}}{2 s}
$$


Therefore, taking $\theta=\left(b(s)-b_{0}\right) / 2 s$ in (5.1) removes the singularity in a natural way. Then one has

$$
\begin{aligned}
\dot{b}(s)\left[\ell e^{b(s)}-k\right] & =-\left(\frac{b(s)+b_{0}}{2 s}\right) \Gamma(b(s), s) \\
& +\frac{1}{s} \int_{b_{0}}^{0}\left[\ell e^{y}-k\right]\left(y-\frac{b(s)+b_{0}}{2}\right) \Gamma(b(s)-y, s) d y \\
& -\int_{0}^{s} \dot{b}(t)\left[\ell e^{b(t)}-k\right]\left(\frac{b(s)-b_{0}}{2 s}-\frac{b(s)-b(t)}{s-t}\right) \Gamma(b(s)-b(t), s-t) d t .
\end{aligned}
$$

In order to avoid the difficulties at $s=0$ in the numerical simulation, our scheme to compute $b(s)$ starts at time $s=\delta$ which is taken small enough to capture the critical behavior of $b(s)$. In practice, $\delta=10^{-12}$. For notational simplicity, we denote

$$
v(s)=l e^{b(s)}-k b(s) ; \quad z(s)=b_{0}-b(s), \text { and } y(t)=b_{0}-b(t) .
$$

The equation (5.2) can be rewritten as

$$
\frac{d v(s)}{d s}=f(b(s), s)+g(b(s), s)+h(z(s), y(t), s, t)
$$

where

$$
\begin{aligned}
& f(x, s):=-\left(\frac{x+b_{0}}{2 s}\right) \Gamma(x, s) \\
& g(x, s):=\frac{1}{s} \int_{b_{0}}^{0} g_{1}(x, s, y) d y \\
& g_{1}(x, s, y):=\left[\ell e^{y}-k\right]\left(y-\frac{x+b_{0}}{2}\right) \Gamma(x-y, s) \\
& h(z(s), y(t), s, t):=\int_{0}^{s} h_{1}(z(s), y(t), s, t) \dot{v}(t) d t \\
& h_{1}(z(s), y(t), s, t):=\left(\frac{z(s)}{2 s}-\frac{z(s)-y(t)}{s-t}\right) \Gamma(y(t)-z(s), s-t)
\end{aligned}
$$

The following iterative scheme is employed to solve the equation (5.3), for iterative index $n \geqslant 1$

$$
\begin{aligned}
\frac{d v^{(n)}}{d s} & =f\left(b^{(n)}(s), s\right)+g\left(b^{(n)}(s), s\right)+h\left(z^{(n-1)}(s), y^{(n-1)}(t), s, t\right) \\
b^{(n)}(\delta) & =b_{\delta}, \quad h\left(z^{(0)}(s), y^{(0)}(t), s, t\right) \equiv 0 .
\end{aligned}
$$

Notice that for each $n$, the equation (5.4) in fact is an ODE which can be solved numerically by standard schemes such as the Euler method or the more accurate Runge-Kutta (RK) 
method, etc. In the $n$th iteration, the function $h\left(z^{(n-1)}(s), y^{(n-1)}(t), s, t\right)$ can be regarded as a known forcing function whose values at all $s$ are already computed in the previous $(n-1)$ th iteration. The left (right) rectangular rule, trapezoid rule or quadrature rule are used to approximate the integrations of $g$ and $h$. More details of the scheme are as follows.

\section{Generating mesh points.}

To better capture the behavior of $b(s)$ in the region of small $s$, a strict monotonic function $s^{(G)}=100 s+\ln s$ is used to generate mesh points which are concentrated near 0 and suitable for our scheme. Let $s_{\min }^{(G)}=s_{1}^{(G)}, s_{2}^{(G)}, \ldots, s_{M+1}^{(G)}=s_{\max }^{(G)}, M \in \mathbb{N}$ be evenly-spaced points of $\left[s_{\text {min }}^{(G)}, s_{\text {max }}^{(G)}\right]$, then $s_{1}=\delta, s_{2}, \ldots, s_{M+1}$ are the grid points for our scheme, where

$$
s_{i}^{(G)}=100 s_{i}+\ln s_{i}, \quad i=1,2, \ldots, M+1
$$

\section{Scheme for (5.4).}

Denote $\Delta s_{i}=s_{i+1}-s_{i}$ and let $v_{i}^{(n)}, b_{i}^{(n)}, z_{i}^{(n)}, y_{i}^{(n)}, F\left(b_{i}^{(n)}, s_{i}\right), G\left(b_{i}^{(n)}, s_{i}\right), H_{i}^{(n)}$ be the approximations of $v^{(n)}\left(s_{i}\right), b^{(n)}\left(s_{i}\right), z^{(n)}\left(s_{i}\right), y^{(n)}\left(s_{i}\right), f\left(b_{i}^{(n)}, s_{i}\right), g\left(b_{i}^{(n)}, s_{i}\right), h\left(b_{i}^{(n)}, b^{(n)}(t), s_{i}, t\right)$ respectively, then the Scheme for (5.4) is

a) Euler

$$
\begin{aligned}
& v_{i+1}^{(n)}=v_{i}^{(n)}+\left[F\left(b_{i}^{(n)}, s_{i}\right)+G\left(b_{i}^{(n)}, s_{i}\right)+H_{i}^{(n-1)}\right] \Delta s_{i}, \quad i=1, \ldots M, \quad n \geqslant 1 \\
& v_{1}^{(n)}=v_{\delta}:=l e^{b_{\delta}}-k b_{\delta}, \quad H_{i}^{(0)} \equiv 0 .
\end{aligned}
$$

where $F\left(b_{i}^{(n)}, s_{i}\right)=f\left(b_{i}^{(n)}, s_{i}\right)$ and $G\left(b_{i}^{(n)}, s_{i}\right), H_{i}^{(n-1)}$ are defined later.

b) Runge-Kutta: to apply the RK method, to generate refined mesh points of $s$ denoted by $s_{1}, s_{1+\frac{1}{2}}, s_{2}, \ldots, s_{M}, s_{M+\frac{1}{2}}, s_{M+1}$. Then $b_{i+\frac{1}{2}}^{(n)}$ is the approximation of $b\left(s_{i+\frac{1}{2}}\right)$ and $H_{i+\frac{1}{2}}^{(n)}$ the approximation of $h\left(b_{i+\frac{1}{2}}^{(n)}, b^{(n)}(t), s_{i+\frac{1}{2}}, t\right)$. Denote $\Delta s_{i+\frac{1}{2}}=s_{i+\frac{1}{2}}-s_{i}$. The RK scheme is

$$
\begin{aligned}
v_{i+1}^{(n)} & =v_{i}^{(n)}+\frac{\Delta s_{i}}{6}\left(K_{1 i}^{(n)}+2 K_{2 i}^{(n)}+2 K_{3 i}^{(n)}+K_{4 i}^{(n)}\right), \quad i=1, \ldots M, \quad n \geqslant 1 ; \\
v_{1}^{(n)} & =v_{\delta}:=l e^{b_{\delta}}-k b_{\delta}, \quad H_{i}^{(0)} \equiv 0 \\
K_{1 i}^{(n)} & =F\left(b_{i}^{(n)}, s_{i}\right)+G\left(b_{i}^{(n)}, s_{i}\right)+H_{i}^{(n-1)} ; \\
K_{2 i}^{(n)} & =F\left(b_{i}^{(n)}+K_{1 i}^{(n)} \Delta s_{i+\frac{1}{2}}, s_{i+\frac{1}{2}}\right)+G\left(b_{i}^{(n)}+K_{1 i}^{(n)} \Delta s_{i+\frac{1}{2}}, s_{i+\frac{1}{2}}\right)+H_{i+\frac{1}{2}}^{(n-1)} ; \\
K_{3 i}^{(n)} & =F\left(b_{i}^{(n)}+K_{2 i}^{(n)} \Delta s_{i+\frac{1}{2}}, s_{i+\frac{1}{2}}\right)+G\left(b_{i}^{(n)}+K_{2 i}^{(n)} \Delta s_{i+\frac{1}{2}}, s_{i+\frac{1}{2}}\right)+H_{i+\frac{1}{2}}^{(n-1)} ; \\
K_{4 i}^{(n)} & =F\left(b_{i}^{(n)}+K_{3 i}^{(n)} \Delta s_{i}, s_{i+1}\right)+G\left(b_{i}^{(n)}+K_{3 i}^{(n)} \Delta s_{i}, s_{i+1}\right)+H_{i+1}^{(n-1)} ;
\end{aligned}
$$




\section{Scheme for the integrals $G, H$.}

In the rhs of the equation (5.3), the two integrations are different. The first one, $g$, is a space integral on $\left[b_{0}, 0\right]$ while the second, $h$, is a time integral on $(0, s)$.

a) Approximation of $g$.

Let $y_{1}=b_{0}, y_{2}, \ldots, y_{N}=0, N \in \mathbb{N}$ be the evenly-spaced points of $\left[b_{0}, 0\right]$ and $\Delta y=$ $\left(-b_{0}\right) / M_{0}$, then

$$
\begin{aligned}
g\left(b_{i}^{(n)}, s_{i}\right) & =\frac{1}{s_{i}} \int_{b_{0}}^{0} g_{1}\left(b_{i}^{(n)}, s_{i}, y\right) d y \\
& \approx \frac{1}{s_{i}} \sum_{j=0}^{N} w_{j} g_{1}\left(b_{i}^{(n)}, s_{i}, s_{j}\right) \Delta y:=G\left(b_{i}^{(n)}, s_{i}\right)
\end{aligned}
$$

where $w_{j}$ are weights for a specific quadrature rule; for example, $w_{0}=1 / 2, w_{j}=1,0<j<$ $N, w_{N}=1 / 2$ for the trapezoidal rule as described earlier.

b) Approximation of $h$.

After we have $v_{i}^{(n)}, b_{i}^{(n)}, 1 \leqslant i \leqslant M+1$ for all points, we can approximate $h$ at $s_{i}$ for $1 \leqslant i \leqslant M$

$$
\begin{aligned}
h\left(z_{i}^{(n)}, y^{(n)}(t), s_{i}, t\right) & =\int_{0}^{s} h_{1}\left(z_{i}^{(n)}, y^{(n)}(t), s, t\right) \dot{v}^{(n)}(t) d t \\
& \approx \sum_{j=1}^{i} w_{i j} h_{1}\left(z_{i}^{(n)}, z_{j}^{(n)}, s_{i}, s_{j}\right)\left(v_{j+1}^{(n)}-v_{j}^{(n)}\right) \\
& :=H_{i}^{(n)},
\end{aligned}
$$

where $w_{i j}$ are weights which, for example for the trapezoidal rule, are defined by

$$
\begin{aligned}
& i=1: \quad w_{i j}=1 \\
& i>1: \quad w_{i 1}=w_{i i}=\frac{1}{2}, \quad w_{i j}=1, \quad \text { for } 1<j<i .
\end{aligned}
$$

Then $H_{i+1 / 2}^{(n)}$ for $1 \leq i \leq M$ can be obtained by interpolating $H_{i}^{(n)}$ on the refined mesh points.

\section{Obtaining $b$ from $v$}

After obtaining $v_{i}^{(n)}$ by using (5.4) in the $n$th iteration, $b_{i}^{(n)}$ has to be recovered from $v_{i}^{(n)}$ for the $(n+1)$ th iteration. Recall that the function $l e^{x}-k x$ is strictly monotonic when $x \leqslant b_{0}$. Therefore, for each $v_{i}^{(n)}$ there exists a unique $b_{i}^{(n)}\left(\leqslant b_{0}\right)$ such that

$$
v_{i}^{(n)}=l e^{b_{i}^{(n)}}-k b_{i}^{(n)}
$$


The bisection method is used to obtain $b_{i}^{(n)}$ from $v_{i}^{(n)}$. The values $b_{0}$ and $b^{*}$ are used for the initial guess of the root for the bisection method, and the error tolerance is set as $10^{-14}$.

\subsection{NUMERICAL RESULTS}

In what follows, we consider the following set of parameters: strike price $E=1$, expiration time $T=1$, volatility $\sigma=0.25$, and interest rate $r=0.05$, while letting the dividend rate $D$ vary. The iterative tolerance for $\left|b^{(n+1)}-b^{(n)}\right|$ is set as $10^{-8}$.

The iteration is rapidly convergent. In general for $D \leq r, 7$ iterations provide a solution of error less than $10^{-8}$ and for $D>r 12$ iterations are sufficient. One solution of $B(t)=$ $E e^{b(s)}$ costs less than 2 minutes of computing time on a PC with $\mathrm{C} 2 \mathrm{D}$ cpu. Figure 1 shows the optimal exercise boundary $B(t)$ in the original $S, t$ variables. Numerical accuracy is demonstrated by the overlap of the curves produced from 400, 800 and 1600 mesh points. Afterwords, the numerical results are produced using 800 mesh points.

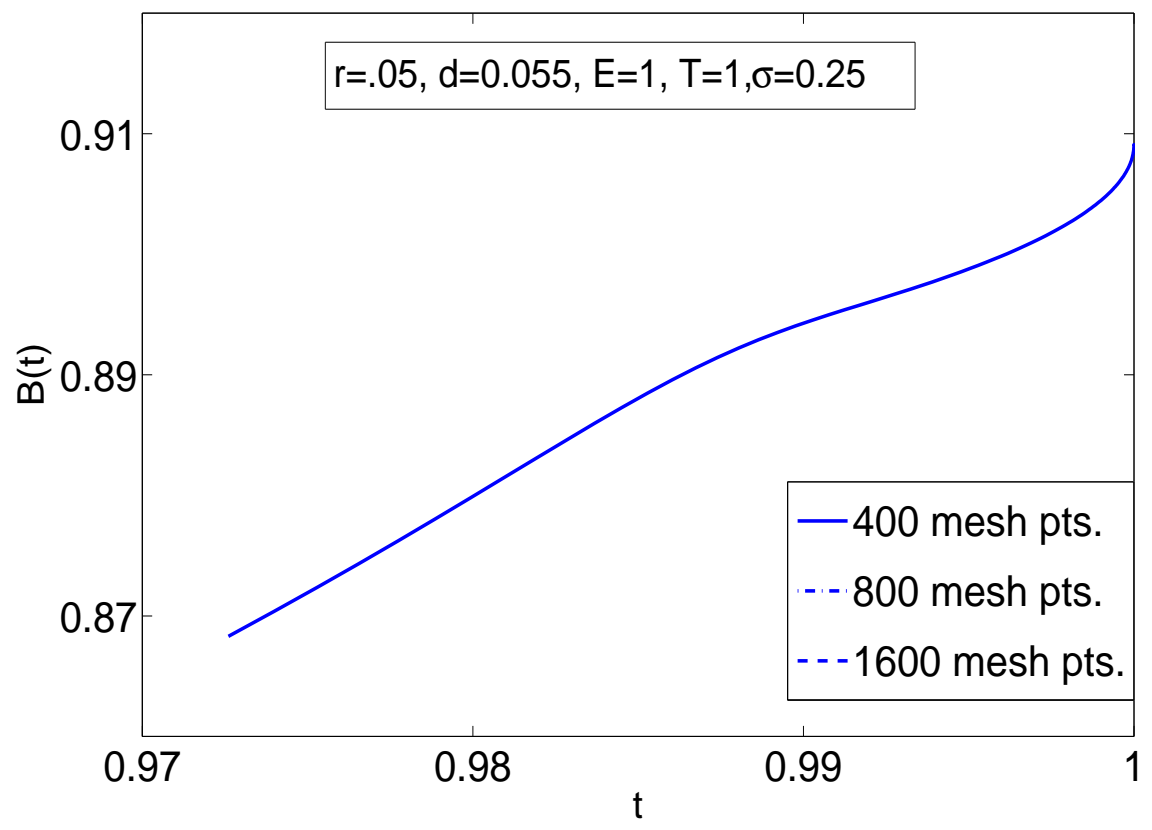

Figure 2: Optimal exercise boundary $B(t)$ 
Figure clearly indicates that the optimal exercise boundary is not convex when $D$ is slightly larger than $r$. Recall that $s=\sigma^{2}(T-t) / 2$ and $b(s)=\ln (B(t) / E)$ so that

$$
B^{\prime \prime}(t)=E \frac{e^{b(s)} \sigma^{2}}{4}\left[\ddot{b}(s)+(\dot{b}(s))^{2}\right]
$$

provides the connection between convexity in the two coordinates. In particular (5.9) says that if $B$ is not convex $\left(B^{\prime \prime}<0\right)$ in some region (as in Figure 2), then $\ddot{b}<0$ is not convex in some $s$-region. On the other hand, if $\ddot{b}>0$ is convex in some $s$-region, then $B$ must be convex in the corresponded t-region.

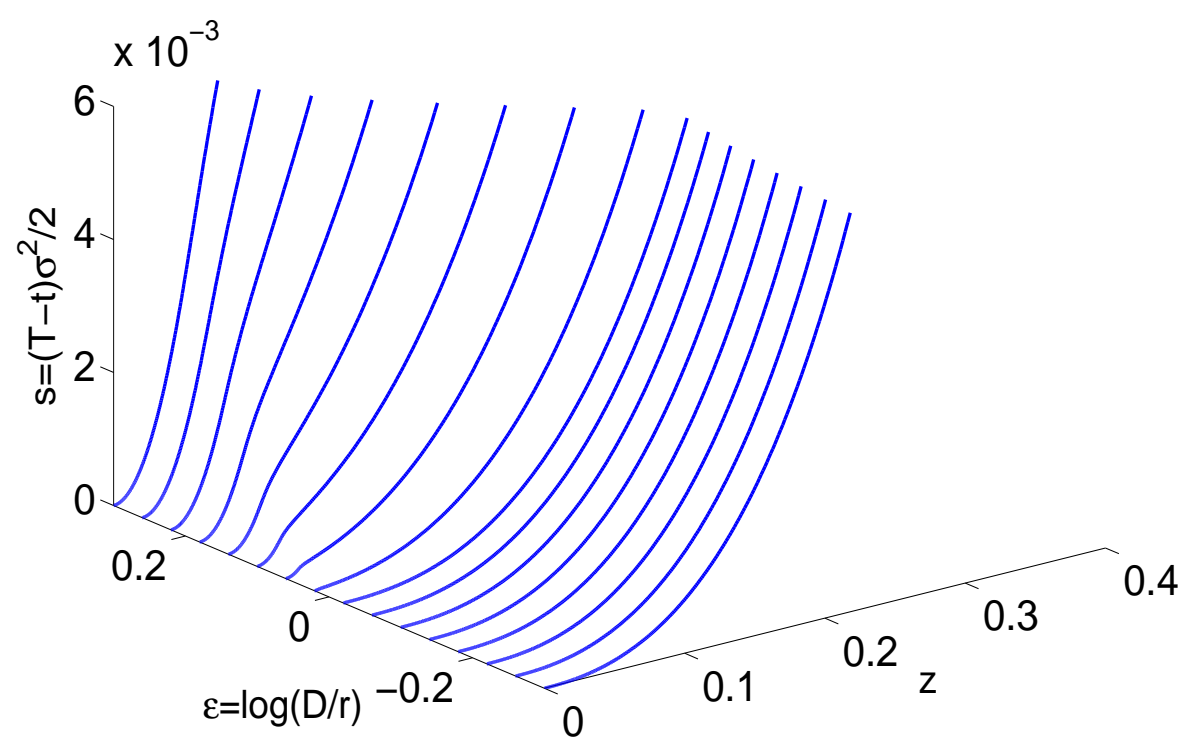

Figure 3: Loss of convexity as $\varepsilon:=\ln (D / r)$ crosses zero.

Figure 3 shows the variation of the free boundary as $\varepsilon=\ln (l / k)=\ln (D / r)$ increases. Here $z=b(0)-b(t)$. For $\varepsilon<0$, all free boundaries $b(s)$ are convex and so are the original optimal exercise boundaries $B(t)$ due to the connection (5.9). For $\varepsilon$ positive and small, the free boundary $b(s)$ loses its convexity near $s=0$, i.e., near expiry. Moreover for $\varepsilon$ positive and increasing, equivalently as $D$ is slightly larger than $r$ and increasing, the non-convex region becomes flatter and spreads out. When $\varepsilon$ or $D$ is large enough, the convexity of $b(s)$ is apparently restored (so is that of $B(t)$ ). 


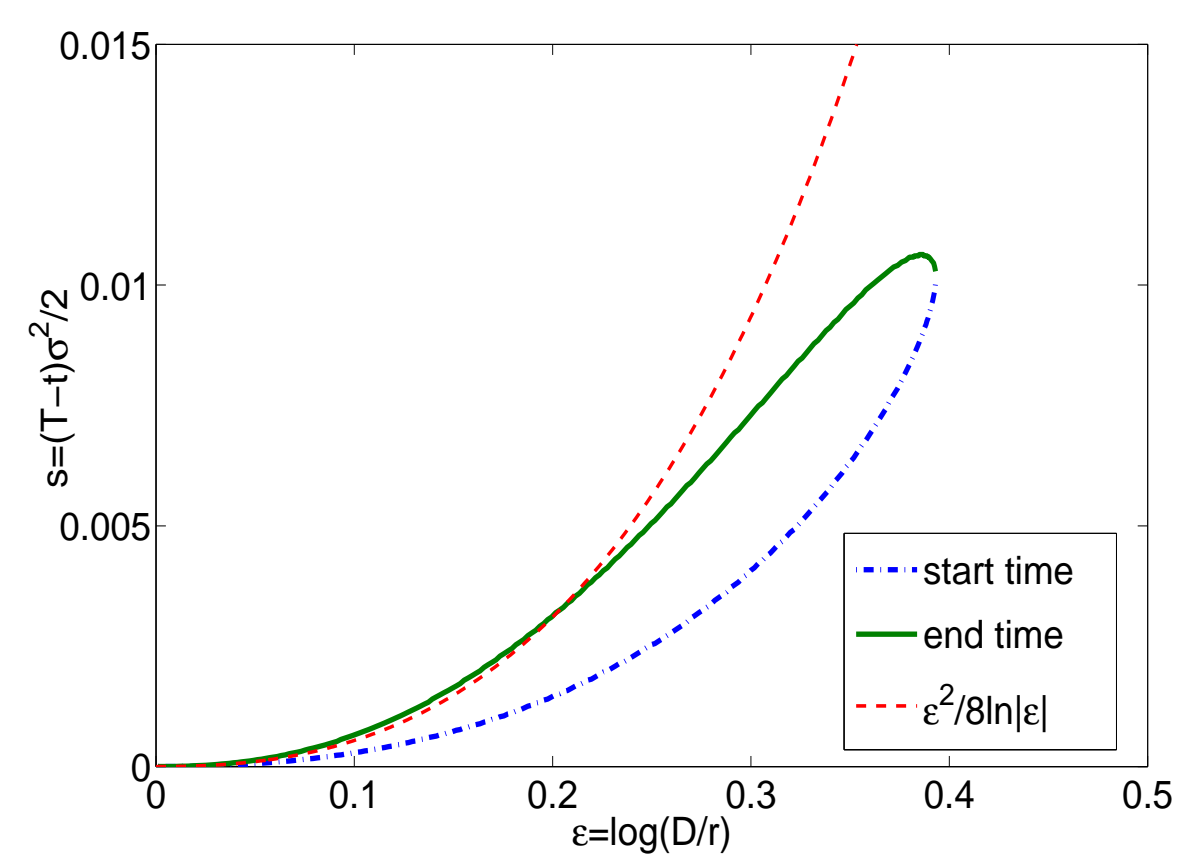

Figure 4: Interval for which the free boundary is not convex

In Figure 4 we plot the location of the onset and termination of the region of nonconvexity for $B(t)$. We obtain a teardrop region which confirms that convexity is lost when $D-r>0$ but is not very large, and that convexity returns when $D$ becomes sufficiently large (e.g., $D>e^{0.4} r \cong 1.5 r$ when $r=0.05$ and the volatility $\sigma=0.25$ ).

Figure 5 shows a plot of the location of the onset and termination of the region of nonconvexity of original $B(t)$ (solid lines) when $\varepsilon$ is very small. The red dashed curve is the analytic estimate for the location of the non-convexity from chapter 6 . 


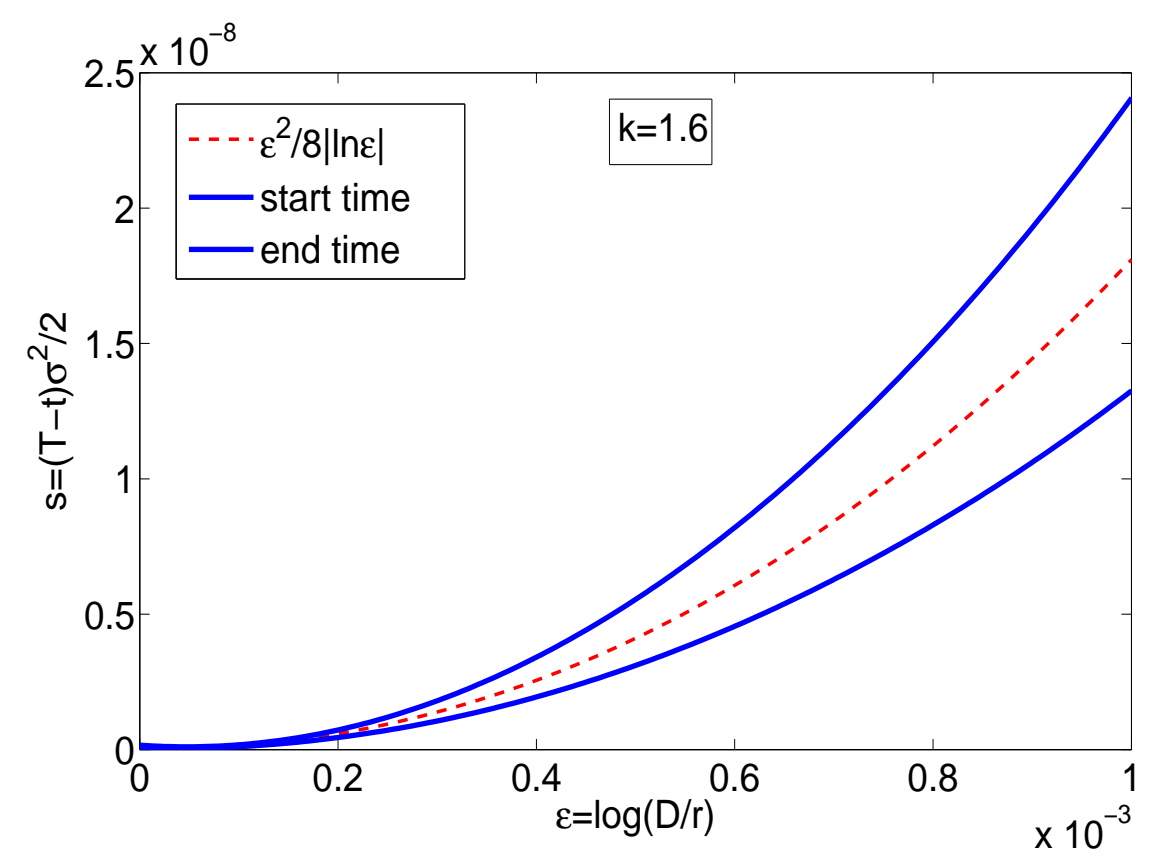

Figure 5: Interval for which the free boundary is not convex 


\subsection{NON-CONVEXITY}

The numerical evidence in the previous chapter suggests that the free boundary $B(t)$ loses convexity when the dividend rate $D$ is larger than the interest rate $r$ but not very large. In this chapter we provide a rigorous proof that $B(t)$ is not convex when $0<D-r \ll 1$, i.e.,

Theorem 6.1. When $0<D-r \ll 1$, the optimal exercise boundary is not convex. More precisely, when $\varepsilon:=\ln (D / r)=\ln (\ell / k)$ is positive and sufficiently small, neither $S=B(t)$ nor $x=b(s)$ is convex. In particular, there exist a $\hat{t}$ for which $B^{\prime \prime}(\hat{t})<0$ and hence $\ddot{b}(\hat{s})<0$, where

$$
0<\hat{s} \leqslant \frac{\varepsilon^{2}}{6|\ln \varepsilon|} \quad \text { and } \quad \hat{t}=T-\frac{2 \hat{s}}{\sigma^{2}}
$$

In this chapter we assume that $\ell>k$.

It is convenient to visualize graphs in the first quadrant, hence consider $z(s)=b_{0}-b(s)$. Also, by the asymptotic analysis in chapter $7, \dot{b}(s)=\nearrow \infty$ when $s \searrow 0$ which motivates us to study the inverse function of $z(s)$. Since $\dot{b}(s)<0$ for $s>0, \dot{z}(s)>0$ for $s>0$. Hence for all $s>0$, there exists an inverse function denoted by $s=s(z)$.

Lemma 6.1. Let $s=s(z)$ be defined as above. If $e^{z} \frac{d s}{d z}$ is not an increasing function, then the original optimal exercise boundary $B(t)$ is not convex. Furthermore, if $B(t)$ is not convex, neither is $s(z)$.

Proof. Recall that

$$
B(t)=E e^{b(s)}=E e^{b_{0}-z(s)} .
$$


It follows from that

$$
\begin{aligned}
\frac{d^{2} B(t)}{d t^{2}} & =\frac{d^{2}\left(e^{b_{0}-z(s)}\right)}{d s^{2}} \\
& =\frac{\sigma^{4} e^{-b_{0}}}{4 E} \frac{d^{2}\left(e^{-z(s)}\right)}{d s^{2}} \\
& =-\frac{\sigma^{4} e^{-b_{0}}}{4 E} \frac{d}{d s}\left(e^{-z(s)} \frac{d z}{d s}\right) \\
& =-\frac{\sigma^{4} e^{-b_{0}}}{4 E} \frac{d}{d z}\left(\left[e^{z} \frac{d s}{d z}\right]^{-1}\right) \frac{d z}{d s} \\
& =\frac{\sigma^{4} e^{-b_{0}}}{4 E}\left(e^{z} \frac{d s}{d z}\right)^{-2} \frac{d}{d z}\left(e^{z} \frac{d s}{d z}\right) \frac{d z}{d s} \\
& =\frac{\sigma^{4} e^{-b_{0}}}{4 E} e^{z}\left(\frac{d z}{d s}\right)^{3} \frac{d}{d z}\left(e^{z} \frac{d s}{d z}\right)
\end{aligned}
$$

Hence, if $e^{z} \frac{d s}{d z}$ is not an increasing function, i.e., $\frac{d}{d z}\left(e^{z} \frac{d s}{d z}\right)<0$, then $B(t)$ is not convex. Furthermore one can obtain that

$$
\begin{aligned}
\frac{d^{2} B(t)}{d t^{2}} & =\frac{\sigma^{4} e^{-b_{0}}}{4 E}\left(e^{z} \frac{d s}{d z}\right)^{-2}\left(e^{z} \frac{d s}{d z}+e^{z} \frac{d^{2} s}{d z^{2}}\right) \frac{d z}{d s} \\
& =\frac{\sigma^{4} e^{-b_{0}}}{4 E} e^{-z}\left(\frac{d z}{d s}\right)^{3}\left(\frac{d^{2} s}{d z^{2}}+\frac{d s}{d z}\right) .
\end{aligned}
$$

So if $B(t)$ is not convex, neither is $s(z)$ because $s^{\prime}>0$.

In the following, we will show that when $l>k$ and $\varepsilon=\ln \frac{l}{k} \ll 1$, then $B(t)$ will lose convexity near expiry. To show that $B$ is not convex $\left(B^{\prime \prime}(t) \geqslant 0\right.$ not true), by lemma 6.1 we only need to show that $e^{z} d s / d z$ is not an increasing function. For this, we compare the values of $e^{z} d s / d z$ at two points:

$$
z_{1}:=z\left(s_{1}\right), s_{1}:=\frac{\varepsilon^{2}}{[8+\nu]|\ln \varepsilon|}, \quad z_{2}:=z\left(s_{2}\right), s_{2}:=\frac{\varepsilon^{2}}{[8-\nu]|\ln \varepsilon|}
$$

where $\nu$ can be any constant in $(0,8)$. For definiteness, we fix $\nu=2$. For convenience, we denote the first non-convex position $z^{*}$ and the first non-convex time $s^{*}$, if they exist. If they are infinite, then $B(t)$ is convex in view of $(6.1)$

We can define

$$
z^{*}:=\sup \left\{z>0 \mid \frac{d^{2} s}{d z^{2}}+\frac{d s}{d z} \geq 0 \text { in }(0, z]\right\} ; \quad s^{*}:=s\left(z^{*}\right)
$$


To prove Theorem 6.1, several integral equations are required for our analysis. With the particular choices of $\theta=0$ and $\theta=\left[b(s)-b_{0}\right] / s$ in the equation (4.11), one has

$$
\begin{aligned}
\dot{b}(s)\left[\ell e^{b(s)}-k\right] & =\frac{-b(s) \Gamma(b(s), s)}{s} \\
& +\frac{1}{s} \int_{b_{0}}^{0}\left[\ell e^{y}-k\right][y-b(s)] \Gamma(b(s)-y, s) d y \\
& +\int_{0}^{s} \dot{b}(t)\left[e^{b(t)-b_{0}}-1\right] \frac{b(s)-b(t)}{s-t} \Gamma(b(s)-b(t), s-t) d t,
\end{aligned}
$$

and

$$
\begin{aligned}
\dot{b}(s)\left[\ell e^{b(s)}-k\right] & =\frac{-b_{0} \Gamma(b(s), s)}{s} \\
& +\frac{1}{s} \int_{b_{0}}^{0}\left[\ell e^{y}-k\right]\left[y-b_{0}\right] \Gamma(b(s)-y, s) d y \\
& -\int_{0}^{s} \dot{b}(t)\left[\ell e^{b(t)}-k\right]\left(\frac{b(s)-b_{0}}{s}-\frac{b(s)-b(t)}{s-t}\right) \Gamma(b(s)-b(t), s-t) d t .
\end{aligned}
$$

Noting that $\ell e^{y}-k=k\left[e^{y-b_{0}}-1\right]$, one can use the change of variable $\tilde{y}=y-b_{0}$ in the first integral and the change of variable $y=b_{0}-b(t)$ in the second integral of (6.4) and (6.5) to obtain

$$
\left[1-e^{-z}\right] \frac{d z}{d s}=I_{1}+I_{2}-I_{3}=J_{1}+J_{2}+J_{3}
$$

where

$$
\begin{aligned}
I_{1} & :=\frac{(\varepsilon+z) \Gamma(-\varepsilon-z, s)}{k s}, \\
I_{2} & :=\frac{1}{s} \int_{0}^{\varepsilon}\left[e^{y}-1\right][z+y] \Gamma(-y-z, s) d y, \\
I_{3} & :=\int_{0}^{z}\left[1-e^{-y}\right]\left(\frac{z-y}{s(z)-s(y)}\right) \Gamma(y-z, s(z)-s(y)) d y, \\
J_{1} & :=\frac{\varepsilon \Gamma(-\varepsilon-z, s)}{k s} \\
J_{2} & :=\frac{1}{s} \int_{0}^{\varepsilon} y\left[e^{y}-1\right] \Gamma(-z-y, s) d y \\
J_{3} & :=\int_{0}^{z}\left[1-e^{-y}\right]\left(\frac{z}{s(z)}-\frac{z-y}{s(z)-s(y)}\right) \Gamma(y-z, s(z)-s(y)) d y .
\end{aligned}
$$


Since it is better to consider the inverse function which is smooth on $[0, \infty)$, the equation (6.6) can be rewritten as

$$
\frac{d s}{d z}=\frac{1-e^{-z}}{I_{1}+I_{2}-I_{3}}=\frac{1-e^{-z}}{J_{1}+J_{2}+J_{3}} .
$$

In the sequel, $\varepsilon=\ln (D / r)=\ln (\ell / k)$ is a small positive constant. We use the standard notation $o(1)$ to denote a generic small quantity that approaches zero as $\varepsilon \searrow 0$. We shall utilize the first equation in (6.7) to estimate the positive lower bound of $d s / d z$. The key here is that all terms $I_{1}, I_{2}$ and $I_{3}$ are positive, so we have the basic estimate

$$
\left(1-e^{-z}\right) \frac{d z}{d s} \leqslant I_{1}+I_{2}, \quad \frac{d s}{d z} \geqslant \frac{1-e^{-z}}{I_{1}+I_{2}}
$$

\section{Proof. 1. Lower Bound of $\mathbf{d s} / \mathbf{d z}$}

First we estimate $I_{1}$. From (6.7), we have

$$
I_{1}=\frac{(\varepsilon+z) \Gamma(-\varepsilon-z, s)}{k s}=\frac{\varepsilon+z}{\sqrt{4 \pi k^{2} s^{3}}} e^{-(\varepsilon+z-\alpha s)^{2} / 4 s-k s}
$$

which implies that

$$
\frac{\partial I_{1}}{\partial z}=\frac{e^{-(\varepsilon+z-\alpha s)^{2} / 4 s}}{\sqrt{4 \pi k^{2} s^{3}}}\left\{1-\frac{(\varepsilon+z-\alpha s)(\varepsilon+z)}{2 s}\right\} .
$$

If $s<\varepsilon^{2} / 2$, since $\alpha=k-l-1<0$,

$$
(\varepsilon+z-\alpha s)(\varepsilon+z) \geq(\varepsilon-\alpha s) \varepsilon>\varepsilon^{2}>2 s .
$$

Hence $\frac{\partial I_{1}}{\partial z}<0$ if $s<\varepsilon^{2} / 2$. Therefore

$$
I_{1} \leq \frac{\varepsilon \Gamma(-\varepsilon, s)}{k s}=\frac{\varepsilon e^{-(\varepsilon+\alpha s)^{2} / 4 s-k s}}{\sqrt{4 \pi k^{2} s^{3}}} \leq \frac{\varepsilon e^{-\varepsilon^{2} / 4 s}}{\sqrt{4 \pi k^{2} s^{3}}} .
$$

To estimate $I_{2}$, since $\alpha=k-l-1<-1$ and $z>0$, when $0 \leqslant y \leqslant \varepsilon=-b_{0}$, one has

$$
\begin{aligned}
\Gamma(-y-z, s) & =\frac{1}{\sqrt{4 \pi s}} \exp \left[-\frac{(y+z)^{2}}{4 s}+\frac{(y+z) \alpha}{2}-\left(k+\frac{\alpha^{2}}{4}\right) s\right] \\
& \leqslant \frac{1}{\sqrt{4 \pi s}} \exp \left[-\frac{(y+z)^{2}}{4 s}-\frac{(y+z)}{2}-\left(k+\frac{\alpha^{2}}{4}\right) s\right] \\
& \leqslant \frac{1}{\sqrt{4 \pi s}} \exp \left[-\frac{(y+z)^{2}}{4 s}-\frac{y}{2}-\left(k+\frac{\alpha^{2}}{4}\right) s\right] .
\end{aligned}
$$


It then follows, noting that for $0 \leq y \leq \varepsilon, 0 \leq e^{y}-1 \leq y e^{y} \leq(y+z) e^{y}$, that

$$
\begin{aligned}
I_{2} & :=\frac{1}{s} \int_{0}^{\varepsilon}\left[e^{y}-1\right][z+y] \Gamma(-y-z, s) d y \\
& \leqslant \frac{1}{\sqrt{4 \pi s^{3}}} \exp \left[-\left(k+\frac{\alpha^{2}}{4}\right) s+\frac{\varepsilon}{2}\right] \int_{0}^{\varepsilon}(y+z)^{2} e^{-(y+z)^{2} /(4 s)} d y \\
& =\frac{1}{\sqrt{4 \pi}} \exp \left[-\left(k+\frac{\alpha^{2}}{4}\right) s+\frac{\varepsilon}{2}\right] \int_{z / \sqrt{s}}^{(z+\varepsilon) / \sqrt{s}} \eta^{2} e^{-\eta^{2} / 4} d \eta \\
& \leqslant \frac{1}{\sqrt{4 \pi}} \exp \left[-\left(k+\frac{\alpha^{2}}{4}\right) s+\frac{\varepsilon}{2}\right] \int_{0}^{\infty} \eta^{2} e^{-\eta^{2} / 4} d \eta \\
& =\exp \left[-\left(k+\frac{\alpha^{2}}{4}\right) s\right] e^{\varepsilon / 2} \\
& \leqslant e^{\varepsilon / 2} \quad \forall s>0 .
\end{aligned}
$$

Combining these estimates, we then obtain

$$
\begin{aligned}
\frac{d s}{d z} & =\frac{1-e^{-z}}{I_{1}+I_{2}-I_{3}} \\
& \geqslant \frac{1-e^{-z}}{I_{1}+I_{2}} \\
& \geqslant \frac{z e^{-z}}{\frac{\varepsilon e^{-\varepsilon^{2} /(4 s)}}{\sqrt{4 \pi k^{2} s^{3}}}+e^{\varepsilon / 2}} \quad \forall s \in\left[0, \frac{\varepsilon^{2}}{2}\right] .
\end{aligned}
$$

2. The Lower Bound of $d s / d z$ on $\left[0, s_{2}^{*}\right]$, where $s_{2}^{*}=\min \left\{s_{2}, s^{*}\right\}$.

(1) First we consider $s \in\left[0, s_{1}\right]$ where $s_{1}=\varepsilon^{2} /(10|\ln \varepsilon|), 0<\varepsilon \ll 1$. When $s \in\left[0, s_{1}\right]$, since $e^{-\varepsilon^{2} /(4 s)} / s^{3}$ is a increasing function, from (6.8) one has

$$
I_{1} \leq \frac{\varepsilon e^{-\varepsilon^{2} / 4 s}}{\sqrt{4 \pi k^{2} s^{3}}} \leq \frac{\varepsilon e^{-\varepsilon^{2} / 4 s_{1}}}{\sqrt{4 \pi k^{2} s_{1}^{3}}}=o(1)
$$

Obviously, $z(s)$ is bounded when $s>0$; say $z \leqslant M, M>0$. When $s \in\left(0, s_{1}\right]$, from (6.9), one has

$$
\frac{d s}{d z} \geqslant \frac{1-e^{-z}}{o(1)+e^{\varepsilon / 2}} \geqslant \frac{z e^{-z}}{o(1)+e^{\varepsilon / 2}} \geqslant \frac{z e^{-z}}{o(1)+1} \geqslant \frac{z e^{-M}}{o(1)+1} .
$$

which implies that $z^{2} \leqslant 2(o(1)+1) e^{M} s=o\left(\varepsilon^{2}\right)$. Hence when $s \in\left[0, s_{1}\right], z(s)=o(\varepsilon)$.

In particular, $z_{1}:=z\left(s_{1}\right)=o(\varepsilon)$. Also if $\varepsilon$ is small, $0<z<1 / 2<1$ which implies $z>z^{2}$ and $1-z>z$. Hence

$$
\frac{d s}{d z} \geqslant \frac{z e^{-z}}{o(1)+1}, \quad \forall z \in\left[0, z_{1}\right]
$$


which further implies that

$$
s \geqslant \frac{z^{2} e^{-z}}{o(1)+1} \quad \forall z \in\left[0, z_{1}\right]
$$

To extend the estimate beyond $s_{1}$, we notice that $s^{\prime \prime}+s^{\prime} \geqslant 0$ implies that $\left(e^{z} s^{\prime}\right)^{\prime} \geqslant 0$ so integrating it over $[x, x+h] \subset\left(0, z^{*}\right]$ one obtains

$$
s^{\prime}(x+h) \geqslant e^{-h} s^{\prime}(x) \quad \text { if } 0<x<x+h \leqslant z^{*} .
$$

(2) With $z_{1}:=z\left(s_{1}\right), s^{*}:=s\left(z^{*}\right), s_{2}^{*}:=\min \left\{s_{2}, s^{*}\right\}$, and $z_{2}^{*}:=z\left(s_{2}^{*}\right)$, we claim that $z_{2}^{*}<2 z_{1}$. Suppose not. Then we take $h=z_{1}$ and integrate $(6.12)$ over $\left[0, z_{1}\right]$ to obtain

$$
s\left(2 z_{1}\right)-s\left(z_{1}\right) \geqslant e^{-z_{1}} s\left(z_{1}\right)
$$

hence

$$
s\left(2 z_{1}\right) \geqslant\left[1+e^{-z_{1}}\right] s\left(z_{1}\right)=[2-o(\varepsilon)] s\left(z_{1}\right) .
$$

However, since $s_{2}=\frac{5}{3} s_{1}$, we obtain

$$
s\left(2 z_{1}\right) \geqslant[2-o(\varepsilon)] s_{1}>s_{2} \geqslant s\left(z_{2}^{*}\right),
$$

contradicting the assumption $z_{2}^{*} \geqslant 2 z_{1}$. Hence, we must have $z_{2}^{*}<2 z_{1}$.

(3) We now consider the lower bound of $d s / d z$ in $\left[s_{1}, s_{2}^{*}\right]$. For any $z \in\left[z_{1}, z_{2}^{*}\right] \subset\left[z_{1}, 2 z_{1}\right]$, letting $h=z-z_{1}$ and $x+h=z$ in (6.12) and using (6.11) leads to

$$
\begin{aligned}
\frac{d s(z)}{d z} & \geqslant e^{-\left(z-z_{1}\right)} \frac{d s\left(z_{1}\right)}{d z} \geqslant e^{-\left(z_{2}^{*}-z_{1}\right)} \frac{d s\left(z_{1}\right)}{d z} \\
& \geqslant e^{-z_{2}^{*}} \frac{d s\left(z_{1}\right)}{d z} \geqslant \frac{e^{-z_{2}^{*} z_{1} e^{-z_{1}}}}{o(1)+1} \\
& =\frac{e^{-z_{1}-z_{2}^{*}} z_{1}}{[o(1)+1] z} z \frac{e^{-z_{1}-z_{2}^{*}}}{[o(1)+1]} \frac{z}{2} \\
& \geqslant \frac{z}{4} .
\end{aligned}
$$

In conclusion, when $\varepsilon$ is positive and sufficiently small,

$$
\frac{d s(z)}{d z} \geqslant \frac{z}{4}, \quad \text { for } 0 \leqslant z
$$


which implies that

$$
s(z) \geqslant \frac{z^{2}}{8}, \quad s(z)-s(y) \geqslant \frac{z^{2}-y^{2}}{8}, \quad \forall 0 \leqslant y \leqslant z \leqslant z_{2}^{*} .
$$

\section{Upper Bounds of $d s / d z$}

The optimal exercise boundary is convex if and only if $s^{*}=\infty$. In what follows, we shall show that when $\varepsilon$ is small positive, $s^{*}<s_{2}$. To do this, we show that the value $e^{z} d s / d z$ at $z_{2}$ is much smaller than at $z_{1}$, so it cannot be an increasing function and therefore the free boundary cannot be convex. In (6.11), we already have a lower bound of $e^{z} d s / d z$ at $z_{1}$ if $s^{*} \geqslant s_{1}$ (if $s^{*}<s_{1}$, the non-convexity is established). Here what we need is an upper bound at $z=z_{2}$. The basic idea is to use the second equation in (6.6).

Estimate of $J_{3}$ :

In case $s^{\prime \prime} \geqslant 0$, it is easy to show that $J_{3}$ is positive. Under the weaker condition $s^{\prime \prime}+s^{\prime} \geqslant 0$ in $\left(0, s^{*}\right]$, we shall show that $J_{3}$ is almost positive. For this purpose, we write

$$
J_{3}=\int_{0}^{z}\left[1-e^{-y}\right] R(z, y) \Gamma(y-z, s(z)-s(y)) d y
$$

where

$$
\begin{aligned}
R(z, y) & :=\frac{z}{s(z)}-\frac{z-y}{s(z)-s(y)} \\
& =\frac{y s(z)-z s(y)}{s(z)[s(z)-s(y)]} \\
& =\frac{z y}{s(z)[s(z)-s(y)]}\left(\frac{s(z)}{z}-\frac{s(y)}{y}\right) \\
& =\frac{z y}{s(z)[s(z)-s(y)]} \int_{y}^{z} \frac{\left(\frac{s(x)}{x}\right)^{\prime} d x}{z y} \frac{s^{\prime}(x) x-s(x)}{x^{2}} d x \\
& =\frac{z y}{s(z)[s(z)-s(y)]} \int_{y}^{z} \frac{\int_{0}^{x} \hat{x} s^{\prime \prime}(\hat{x}) d \hat{x}}{x^{2}} d x \\
& =
\end{aligned}
$$


Since $s^{\prime \prime}+s^{\prime} \geqslant 0$ in $\left(0, z^{*}\right]$, when $\hat{x} \in\left(0, z^{*}\right]$, we have $s^{\prime \prime}(\hat{x}) \geqslant-s^{\prime}(\hat{x})$. Also by (6.12), $s^{\prime}(\hat{x}) \leqslant e^{x-\hat{x}} s^{\prime}(x) \leqslant e^{z} s^{\prime}(x)$ when $0<\hat{x} \leqslant x \leqslant z \leqslant z^{*}$. Hence $s^{\prime \prime}(\hat{x}) \geqslant-s^{\prime}(\hat{x}) \geqslant-e^{z} s^{\prime}(x)$. Thus, when $0<y<z<z_{2}^{*}$,

$$
R(z, y) \geqslant-\frac{z y}{s(z)[s(z)-s(y)]} \int_{y}^{z} \frac{\int_{0}^{x} \hat{x} e^{z} s^{\prime}(x) d \hat{x}}{x^{2}} d x=-\frac{z y e^{z}}{2 s(z)} .
$$

Next using (6.13) we derive that

$$
\Gamma(y-z, s(z)-s(y))<\frac{\sqrt{2}}{\sqrt{4 \pi[s(z)-s(y)]}} \leqslant \frac{\sqrt{2}}{\sqrt{\pi\left[z^{2}-y^{2}\right]}} .
$$

Hence,

$$
\begin{aligned}
J_{3} & =\int_{0}^{z}\left[1-e^{-y}\right] R(z, y) \Gamma(y-z, s(z)-s(y)) d y \\
& \geqslant-\int_{0}^{z} y \frac{z y e^{z}}{2 s(z)} \frac{\sqrt{2}}{\sqrt{\pi\left(z^{2}-y^{2}\right)}} d y \\
& =-\frac{\sqrt{\pi} z^{3} e^{z}}{4 \sqrt{2} s(z)} \geqslant-z, \quad \forall z \in\left(0, z_{2}^{*}\right] .
\end{aligned}
$$

Estimate of $J_{2}$ :

One can estimate the lower bound of $J_{2}$ as follows:

$$
\begin{aligned}
J_{2} & =\frac{1}{s} \int_{0}^{\varepsilon} y\left[e^{y}-1\right] \Gamma(-z-y, s) d y \\
& \geqslant \frac{e^{\alpha z-\left(k+\alpha^{2} / 4\right) s}}{\sqrt{4 \pi s^{3}}} \int_{0}^{\varepsilon} y^{2} e^{-(z+y)^{2} /(4 s)} d y \\
& =\frac{e^{\alpha z-\left(k+\alpha^{2} / 4\right) s}}{\sqrt{4 \pi}} \int_{z / \sqrt{s}}^{(z+\varepsilon) / \sqrt{s}} e^{-\eta^{2} / 4} d \eta .
\end{aligned}
$$

In view of (6.13), we see that when $s \in\left(0, s_{2}^{*}\right]$, we have

$$
J_{2} \geqslant \frac{e^{o(\varepsilon)}}{\sqrt{4 \pi}} \int_{2}^{\varepsilon / \sqrt{s}} e^{-\eta^{2} / 4} d \eta \geqslant \frac{1}{4} \int_{2}^{3} e^{-\eta^{2} / 4} d \eta:=c>0 .
$$

It then follows that

$$
J_{2}+J_{3} \geqslant c-z>0 \quad \forall s \in\left(0, s_{2}^{*}\right] \quad\left(\Leftrightarrow \forall z \in\left(0, z_{2}^{*}\right]\right) .
$$


4. Completion of the Proof Now suppose $z^{*}>z_{2}$. Then $z_{2}^{*}=z_{2}$ and $s_{2}^{*}=s_{2}$. Hence we can use the second equation in (6.6) to conclude that

$$
\begin{aligned}
\left.\frac{1}{z} \frac{d s(z)}{d z}\right|_{z=z_{2}} & =\left.\frac{1-e^{-z}}{z} \frac{1}{J_{1}+J_{2}+J_{3}}\right|_{z=z_{2}} \\
& \leqslant\left.\frac{1}{J_{1}}\right|_{z=z_{2}} \\
& =\left.\frac{k}{\varepsilon} \sqrt{4 \pi s^{3}} e^{(\varepsilon+z(s)-\alpha s)^{2} /(4 s)+k s}\right|_{s=s_{2}=\varepsilon^{2} /(-6 \ln \varepsilon)} \leqslant \varepsilon^{1 / 4}
\end{aligned}
$$

In comparing with (6.13), we see that

$$
\begin{aligned}
e^{z_{2}} \frac{d s\left(z_{2}\right)}{d z}-e^{z_{1}} \frac{d s\left(z_{1}\right)}{d z} & \leqslant z_{2} e^{z_{2}} \varepsilon^{1 / 4}-\frac{1}{2} e^{z_{1}} z_{1} \\
& \leqslant 2 z_{1} e^{2 z_{1}} \varepsilon^{1 / 4}-\frac{e^{z_{1}} z_{1}}{2}<0 .
\end{aligned}
$$

This implies that $e^{z} d s(z) / d z$ is not an increasing function on $\left[z_{1}, z_{2}\right]$. Consequently, we must have $z^{*}<z_{2}$. This completes the proof of Theorem 6.1 . 


\subsection{NEAR-EXPIRY BEHAVIOR}

Near-expiry estimates for $b(s)$ and $B(t)=E e^{b(s)}$ have been obtained using asymptotic analysis for the entire range of the parameters $D$ and $r[32,42]$. The leading order expansion can be made rigorous. A rigorous proof for $b(s)$ alone can be found in [34] and another for $D=0$

appears in [15]. Here we are more interested in the estimate for $\ddot{b}(s)$ which implies that very near expiry, the optimal exercise boundary begins convex. The location of the non-convex region occurs a little farther from expiry near $\hat{t}$ given in Theorem 6.1. We show that

Theorem 7.1. Assume that $D>r$. Let $A=0.903446597884 \ldots$. Then

$$
b(s)=\ln \frac{r}{D}-[A+o(1)] \sqrt{s}, \quad \dot{b}(s)=-\frac{A+o(1)}{2 \sqrt{s}}, \quad \ddot{b}(s)=\frac{A+o(1)}{4 s^{3 / 2}} \quad \forall s>0
$$

where $\lim _{s \downarrow 0} o(1)=0$. Consequently, the inverse, $s=s(z)$, of $z=\ln (D / r)-b(s)$ satisfies

$$
s(z)=\frac{z^{2}}{A^{2}+o(1)}, \quad s^{\prime}(z)=\frac{2 z}{A^{2}+o(1)}, \quad s^{\prime \prime}(z)=\frac{2}{A^{2}+o(1)} \quad \forall z \geqslant 0 .
$$

Proof. Instead of considering the premium $\phi=p-p_{0}$, we consider the rate, $q=p_{s}=\phi_{s}$, of the premium change. We study the family $\left\{q^{L}, b^{L}\right\}_{L>0}$ which magnifies the region of interest near $s=0$ and is defined by

$$
q^{L}(x, s):=L q\left(b_{0}+L^{-1} x, L^{-2} s\right), \quad b^{L}(s)=L\left[b\left(L^{-2} s\right)-b_{0}\right] .
$$

Information obtained from $b^{L}$ will be cycled back to $b$ through the identity

$$
b(s \theta)=b_{0}+\left.\sqrt{s} b^{L}(\theta)\right|_{L=1 / \sqrt{s}} \quad \forall \theta \in\left[\frac{1}{2}, 2\right], s>0 .
$$

We shall show that $\lim _{L \rightarrow \infty} b^{L}(\theta)=A_{1} \sqrt{\theta}$ in $C^{2}([\delta, h])$ for any $h>\delta>0$. 
For each $L>0$, by direct substitution into (3.4) and (3.5) $\left(q^{L}, b^{L}\right)$ satisfies

$$
\begin{cases}q_{s}^{L}=q_{x x}^{L}+\alpha L^{-1} q_{x}^{L}-k L^{-2} q^{L} & \forall x>b^{L}(s), s>0, \\ q^{L}(x, s)=0 & \forall x \leqslant b^{L}(s), s>0, \\ k L\left[e^{L^{-1} b^{L}(s)}-1\right] \dot{b}^{L}(s)=q_{x}^{L}\left(b^{L}(s), s\right) & \forall s>0, \\ q^{L}(x, 0)=\delta(x-\varepsilon L)+k \max \left\{\frac{e^{x / L}-1}{L}, 0\right\} & \forall x \in \mathbb{R} .\end{cases}
$$

From (3.12) we derive that

$$
\begin{gathered}
0<b^{L}(s) \dot{b}^{L}(s) \leqslant C, \quad 0>b^{L}(s) \geqslant-\sqrt{2 C s} \quad \forall s>0, \\
0 \leqslant q^{L}(x, s)=L q\left(b_{0}+x L^{-1}, s L^{-2}\right) \leqslant L C\left\{b_{0}+x L^{-1}-b\left(L^{-2} s\right)\right\} \\
=C\left[x-b^{L}(s)\right] \leqslant C[x+\sqrt{2 C s}] \quad \forall s>0, x \in\left[b^{L}(s), \varepsilon L / 2\right] .
\end{gathered}
$$

Since the bounds of the above estimates for $q^{L}$ and $b^{L}$ are independent of $L$, one can show that $\left\{\left(q^{L}, b^{L}\right)\right\}_{L>1}$ is locally compact and we can select a subsequence along which $\left(q^{L}, b^{L}\right)$ approaches a limit, $(\Psi, \zeta)$. The limit satisfies

$$
\begin{cases}\Psi_{s}=\Psi_{x x} & \forall x>\zeta(s), s>0, \\ \Psi(x, s)=0, & \forall x \leqslant \zeta(s), s>0, \\ k \zeta(s) \dot{\zeta}(s)=\Psi_{x}(\zeta(s), s) & \forall s>0, \\ \Psi(x, 0)=k \max \{0, x\} & \forall x \in \mathbb{R}, \\ 0 \leqslant \Psi(x, s) \leqslant C[x-\zeta(s)] & \forall x \geqslant \zeta(s), s \geqslant 0 .\end{cases}
$$

Since the solution is unbounded, the last condition imposes a constraint on the growth of the solution.

This problem admits a unique solution and the solution is self-similar, given by

$$
\begin{aligned}
\zeta(s) & =A_{1} \sqrt{s} \quad \forall s>0, \\
\Psi(x, s) & =k\left(x-\frac{A_{1} \sqrt{s} \int_{x / \sqrt{s}}^{\infty}\left(\eta-\frac{x}{\sqrt{s}}\right) e^{-\eta^{2} / 4} d \eta}{\int_{A_{1}}^{\infty}\left(\eta-A_{1}\right) e^{-\eta^{2} / 4}}\right) \quad \forall x \geqslant \zeta(s), s>0,
\end{aligned}
$$


where $A_{1}$ is the solution of the equation

$$
1+\frac{A_{1} \int_{A_{1}}^{\infty} e^{-\eta^{2} / 4} d \eta}{\int_{A_{1}}^{\infty}\left(\eta-A_{1}\right) e^{-\eta^{2} / 4} d \eta}=\frac{A_{1}^{2}}{2}
$$

It is easy to verify that this equation is equivalent to that appearing in [42].

Once we know the uniqueness of the limit, we then know that the whole sequence $\left(q^{L}, b^{L}\right)$ converges. In addition, by compactness, $\lim _{L \rightarrow \infty} b^{L}=\zeta$ in $C^{2}([1 / 2,2])$. Using $(7.1)$ and its differentiation with respect to $\theta$, we obtain the assertion of Theorem 7.1. This completes the proof.

In [42], the ideas were presented for obtaining a formal estimate for the leading order behavior of $b(s)$ in Theorem 7.1. Here we extend these ideas to a full expansion and provide two alternative leading order expansions of $b(s)$.

1. A Formal Expansion. Recall that $\phi:=p-p_{0}$ satisfies $\phi_{s}-\mathcal{L} \phi=\delta(x)+k\left[e^{x-b_{0}}-1\right] H(x-$ $b(t)) H(-x)$, with zero initial value. When $\varepsilon=-b_{0}=\ln (\ell / k)>0$, the Delta function will not interfere much with the solution near $\left(b_{0}, 0\right)$. Hence, we expect the following expansion

$$
\phi(x, s)=\left.\Phi(\xi, s)\right|_{\xi=\frac{x-b(0)}{\sqrt{s}}}, \quad \Phi(\xi, s) \sim s \sum_{n=1}^{\infty} \phi_{n}(\xi) s^{n / 2}, \quad b(s) \sim b_{0}+\sum_{n=1}^{\infty} A_{n} s^{n / 2}
$$

Note that $\Phi$ satisfies

$$
\Phi_{s}-\frac{\xi}{2 s} \Phi_{\xi}-\frac{1}{s} \Phi_{\xi \xi}=\frac{\alpha}{\sqrt{s}} \Phi_{\xi}-k \Phi+k \sum_{n=1}^{\infty} \frac{\xi^{n} s^{n / 2}}{n !} \quad \forall \xi \in\left(\frac{b(s)+\varepsilon}{\sqrt{s}}, \frac{\varepsilon}{\sqrt{s}}\right), s>0
$$

This leads to the following

$$
\mathcal{L}_{n} \phi_{n}=\frac{k \xi^{n}}{n !}+\alpha \phi_{n-1}-k \phi_{n-2} \quad \forall \xi \in\left(A_{1}, \infty\right)
$$

where

$$
\mathcal{L}_{n} \psi:=\left(1+\frac{n}{2}-\frac{\xi}{2} \frac{d}{d \xi}-\frac{d^{2}}{d \xi^{2}}\right) \psi
$$

Here we have used the extension $\phi_{0}=\phi_{-1} \equiv 0$. 
The boundary conditions for $\phi_{n}$ and the unknown $A_{n}$ will be derived from

$$
\begin{gathered}
0=\phi(b(s), s)=\Phi(\xi(s), s), \quad 0=\phi_{x}(b(s), s)=s^{-1 / 2} \Phi_{\xi}(\xi(s), s), \\
\xi(s)=\left[b(s)-b_{0}\right] s^{-1 / 2} \sim A_{1}+\sum_{n=2}^{\infty} A_{n} s^{n-1 / 2} .
\end{gathered}
$$

Using the above asymptotic expansion, we have

$$
\begin{aligned}
0 & \sim \sum_{n=1}^{\infty} s^{n / 2} \phi_{n}\left(A_{1}+\sum_{m=2}^{\infty} A_{m} s^{(m-1) / 2}\right) \\
& \sim \sum_{n=1}^{\infty} s^{n / 2} \sum_{i=0}^{\infty} \frac{\phi_{n}^{(i)}\left(A_{1}\right)}{i !}\left(\sum_{m=2}^{\infty} A_{m} s^{(m-1) / 2}\right)^{i} \\
& \sim \phi_{1}\left(A_{1}\right) s^{1 / 2}+\left[\phi_{2}\left(A_{1}\right)+\phi_{1}^{\prime}\left(A_{1}\right) A_{2}\right] s+\sum_{n=3}^{\infty}\left[\phi_{n}\left(A_{1}\right)+\phi_{1}^{\prime}\left(A_{1}\right) A_{n}+\cdots\right] s^{n / 2} \\
0 & \sim \sum_{n=1}^{\infty} s^{n / 2} \phi_{n}^{\prime}\left(A_{1}+\sum_{m=2}^{\infty} A_{m} s^{(m-1) / 2}\right) \\
& \sim \phi_{1}^{\prime}\left(A_{1}\right) s^{1 / 2}+\left[\phi_{2}^{\prime}\left(A_{1}\right)+\phi_{1}^{\prime \prime}\left(A_{1}\right) A_{2}\right] s+\sum_{n=3}^{\infty}\left[\phi_{n}^{\prime}\left(A_{1}\right)+\phi_{1}^{\prime \prime}\left(A_{1}\right) A_{n}+\cdots\right] s^{n / 2} .
\end{aligned}
$$

Hence, we obtain the boundary conditions and the free boundary conditions

$$
\begin{array}{lll}
\phi_{1}\left(A_{1}\right)=0, & \phi_{1}^{\prime}\left(A_{1}\right)=0, & \phi_{1}(\xi)=O(\xi) \text { as } \xi \rightarrow \infty, \\
\phi_{2}\left(A_{1}\right)=0, & \phi_{2}^{\prime}\left(A_{1}\right)+\phi_{1}^{\prime \prime}\left(A_{1}\right) A_{2}=0, & \phi_{2}(\xi)=O\left(\xi^{2}\right) \text { as } \xi \rightarrow \infty, \\
\phi_{n}\left(A_{1}\right)=a_{n-1}, & \phi_{n}^{\prime}\left(A_{1}\right)+\phi_{1}^{\prime \prime}\left(A_{1}\right) A_{n}=b_{n-1}, & \phi_{n}(\xi)=O\left(\xi^{n}\right) \text { as } \xi \rightarrow \infty
\end{array}
$$

where $a_{m}, b_{m}$ are constants depending only expansions of order up to $m$.

For the homogeneous equation $\mathcal{L}_{n} \psi=0$, one can verify that the following are two linear independent solutions:

$$
\psi_{n}(\xi)=\int_{\xi}^{\infty}(\eta-\xi)^{n+2} e^{-\eta^{2} / 4} d \eta, \quad \tilde{\psi}_{n}(\xi)=\int_{\mathbb{R}}(\eta-\xi)^{n+2} e^{-\eta^{2} / 4} d \eta
$$

Here $\tilde{\psi}_{n}$ is a polynomial of degree $n+2$. It is easy to verify that

$$
\phi_{1}(\xi)=k\left\{\xi-\frac{A_{1} \psi_{1}(\xi)}{\psi_{1}\left(A_{1}\right)}\right\}
$$


where $A_{1}$ is the solution of the transcendental equation

$$
\int_{A_{1}}^{\infty}\left(\eta-A_{1}\right)^{2}\left(\eta+2 A_{1}\right) e^{-\eta^{2} / 4} d \eta=0 \quad \Rightarrow \quad A_{1}=-0.9034465978843 \ldots
$$

2. An Alternative Formal Derivation We can also use the first or second equation in (6.6) to derive the asymptotic behavior. Assume that $0<s<\varepsilon^{3}$. Also assume that $z(s)=[A+O(\sqrt{s})] \sqrt{s}$. Then one finds

$$
\begin{aligned}
J_{1} & \leqslant \frac{-b_{0}}{k \sqrt{4 \pi} s^{3 / 2}} \exp \left[-\frac{\left(-b_{0}-\alpha s\right)^{2}}{4 s}-k s\right] \\
& \leqslant \frac{-b_{0}}{k \sqrt{4 \pi} s^{3 / 2}} \exp \left(-\frac{b_{0}^{2}}{4 s}\right) \leqslant O\left(\frac{\exp \left(-b_{0}^{2} / \varepsilon^{3}\right)}{\varepsilon^{9 / 2}}\right) .
\end{aligned}
$$

Hence $J_{1}$ can be neglected in the expansion for small $s$. Also,

$$
J_{2}=\frac{1}{\sqrt{4 \pi}} \int_{A}^{\infty}(A-\eta)^{2} e^{-\eta^{2} / 4} d \eta+O(z) .
$$

For $J_{3}$, one uses the change of variable

$$
\eta=\frac{(z-y)}{\sqrt{s(z)-s(y)}} \approx A \sqrt{\frac{z-y}{z+y}} .
$$

Then one derives that

$$
J_{3}=\frac{A^{2}}{\sqrt{4 \pi}} \int_{0}^{A}\left(\frac{A^{2}-\eta^{2}}{A^{2}+\eta^{2}}\right)^{2} e^{-\eta^{2} / 4} d \eta+O(z) .
$$

Thus the differential equation (6.6) gives the following equation for $A$ :

$$
\begin{gathered}
A^{2}=\frac{1}{\sqrt{\pi}} \int_{A}^{\infty}(A-\eta)^{2} e^{-\eta^{2} / 4} d \eta+\frac{A^{2}}{\sqrt{\pi}} \int_{0}^{A}\left(\frac{A^{2}-\eta^{2}}{A^{2}+\zeta^{2}}\right)^{2} e^{-\eta^{2} / 4} d \eta \\
\Longrightarrow \quad A=0.9034465978843 \ldots
\end{gathered}
$$

Numerically it is evident that $A=-A_{1}$ and is precisely the value obtained in [42]. 


\subsection{FAR-FROM EXPIRY BEHAVIOR}

The numerical simulations in chapter 5 suggest that as the dividend rate $D$ grows larger than $r$, the non-convex region broadens (and flattens) and moves farther from expiry. Indeed, for large enough $D$, the numerics suggest that convexity is restored. We anticipate that a rigorous analysis of this phenomenon will require sharp estimates on the far-from-expiry behavior of the American put option of the sort summarized in this chapter.

Let $(p, b)$ be the solution of the variational problem (2.6) and (2.7). We are interested in the behavior of $b(s)$ and $p(x, s)$ as $s \rightarrow \infty$. From chapter 3 , we have

$$
p(\cdot, s) \nearrow p^{*}(\cdot), \quad b(s) \searrow b^{*} \quad \text { as } s \nearrow \infty
$$

where $\left(p^{*}, b^{*}\right)$ is the solution of the infinite horizon problem, given by

$$
p^{*}(x):=\max \left\{1-e^{x}, \frac{e^{-\lambda\left(x-b^{*}\right)}}{1+\lambda}\right\}, \quad b^{*}:=\ln \frac{\lambda}{1+\lambda}, \quad \lambda:=\frac{\alpha}{2}+\sqrt{\beta}
$$

In this chapter, we prove the following,

Theorem 8.1. There exists a constant $m>0$ such that for each $s \geqslant 1$,

$$
b(s)=b^{*}+\left[m+O(1) s^{-\frac{1}{2}}\right] s^{-\frac{3}{2}} e^{-\beta s}, \quad \dot{b}(s)=-\left[m \beta+O(1) s^{-\frac{1}{2}}\right] s^{-\frac{3}{2}} e^{-\beta s},
$$

where $O(1)$ is a generic function bounded uniformly in $s \in[1, \infty)$. 
Theorem 8.2 (Decay Rates). There exist positive constants $c$ and $C$ such that for each $s \geqslant 1$,

$$
c \rho(s) \leqslant\left\|p(\cdot, s)-p^{*}(\cdot)\right\|_{L^{\infty}(\mathbb{R})} \leqslant C \rho(s), \quad c \rho(s) \leqslant\left\|p_{s}(\cdot, s)\right\|_{L^{\infty}(\mathbb{R})} \leqslant C \rho(s)
$$

where

$$
\rho(s)= \begin{cases}s^{-\frac{3}{2}} e^{-\beta s} & \text { if } \alpha>0, \\ s^{-1} e^{-k s} & \text { if } \alpha=0, \\ s^{-\frac{1}{2}} e^{-k s} & \text { if } \alpha<0 .\end{cases}
$$

Theorem 8.3 (Asymptotic Profiles). There exist constants $c_{1}>0$ and $c_{2} \in \mathbb{R}$ that depend only on $k$ and $\ell$ such that for every $x \in \mathbb{R}$ and $s \geqslant 2$,

$$
\frac{p_{s}(x, s)}{\rho(s)}= \begin{cases}e^{-\xi^{2}}\left\{c_{1}+c_{2} \xi s^{-\frac{1}{2}}+O(1)\left(1+\xi^{2}\right) s^{-1}\right\} & \text { if } \alpha<0, \\ z^{+} e^{-[z+b(s) / \sqrt{4 s}]^{2}}\left\{c_{1}+O(1)(1+z \ln s) s^{-1 / 2}\right\} & \text { if } \alpha=0, \\ {[x-b(s)]^{+} e^{-x^{2} /(4 s)-\alpha x / 2}\left\{c_{1}+O(1)\left(s^{-1 / 2}+|x| s^{-1} \ln s\right)\right\}} & \text { if } \alpha>0\end{cases}
$$

where $\xi:=(x+\alpha s) / \sqrt{4 s}, z:=(x-b(s)) / \sqrt{4 s}, z^{+}:=\max \{0, z\}$, and $O(1)$ is a function bounded uniformly in $(x, s) \in \mathbb{R} \times[1, \infty)$. Consequently, for some positive constant $c$ depending on $k$ and $\ell$,

$$
\lim _{s \rightarrow \infty}\left\|\frac{p^{*}-p}{\rho(s)}-\Psi\right\|_{L^{\infty}(\mathbb{R})}=0 ; \quad \Psi(x, s):= \begin{cases}c e^{-\xi^{2}} & \text { if } \alpha<0, \\ c z^{+} e^{-z^{2}} & \text { if } \alpha=0, \\ c\left[x-b^{*}\right]^{+} e^{-\alpha x / 2} & \text { if } \alpha>0 .\end{cases}
$$


Proof of Theorem 8.1. In the case $\ell=0$ (i.e. no dividend), Chen and Chadam already provided an outline of the proof in [15]. Here we follow that outline and provide the full details for the general case $\ell \geqslant 0$.

Choosing $\theta=b(s) / s$ in identity (4.11) and dividing (4.9) and (4.11) by $\Gamma(b(s), s)$ and $\Gamma(b(s), s) / s$, respectively, one obtains the following integral identities that are valid for all $\ell \geq 0$ :

$$
\begin{gathered}
1+\int_{b_{0}}^{0} A(s, y) d y=\int_{0}^{s} B(s, t) d t \\
\frac{\dot{b}(s)\left[\ell e^{b(s)}-k\right] s}{\Gamma(b(s), s)}=\int_{b_{0}}^{0} y A(s, y) d y+\int_{0}^{s}[t \delta(s, t)-b(t)] B(s, t) d t
\end{gathered}
$$

where, for $s>0, y \in\left(b_{0}, 0\right), t \in(0, s)$,

$$
\begin{aligned}
A(s, y) & :=\left[\ell e^{y}-k\right] \frac{\Gamma(b(s)-y, s)}{\Gamma(b(s), s)}=A_{\infty}(y) e^{\delta_{1}(s, y)}, \\
B(s, t) & :=\dot{b}(t)\left[\ell e^{b(t)}-k\right] \frac{\Gamma(b(s)-b(t), s-t)}{\Gamma(b(s), s)}=\zeta(t)\left(1-\frac{t}{s}\right)^{-1 / 2} e^{\delta_{2}(s, t)} \\
A_{\infty}(y) & :=\left[\ell e^{y}-k\right] e^{\alpha y / 2} \\
\zeta(t) & :=\dot{b}(t)\left[\ell e^{b(t)}-k\right] e^{\beta t+\alpha b(t) / 2} \\
\delta(s, t) & :=\frac{b(s)-b(t)}{s-t} \\
\delta_{1}(s, y) & :=\frac{2 b(s) y-y^{2}}{4 s} \\
\delta_{2}(s, t) & :=\frac{b^{2}(s)}{4 s}-\frac{[b(s)-b(t)]^{2}}{4(s-t)} .
\end{aligned}
$$

Note that the terms $\int_{b_{0}}^{0} A d y$ and $\int_{b_{0}}^{0} y A d y$ do not appear when $\ell \leqslant k$ since $b_{0}=0$.

In chapter 3 we showed that

$$
b \in C^{\infty}((0, \infty)), \quad \lim _{s \searrow 0} b(s)=b_{0}:=\min \{0, \ln (k / \ell)\}, \quad \dot{b}(s)<0, \ell e^{b(s)}-k<0 \quad \forall s>0 .
$$

Noting that $A>0, B>0, \delta<0$, it then follows from (8.1) and (8.2) that for every $s \geqslant 1$,

$$
\begin{aligned}
0<\frac{\dot{b}(s)\left[\ell e^{b(s)}-k\right] s}{\Gamma(b(s), s)} & \leqslant \int_{0}^{s}[t \delta(s, t)-b(t)] B(s, t) d t \leqslant \int_{0}^{s}[-b(t)] B(s, t) d t \\
& \leqslant|b(s)| \int_{0}^{s} B(s, t) d t=|b(s)|\left\{1+\int_{b_{0}}^{0} A(s, y) d y\right\} \\
& \leqslant\left|b^{*}\right|\left\{1+\int_{b_{0}}^{0}\left[l e^{y}-k\right] e^{\alpha y / 2} e^{\left(2 b(s) y-y^{2}\right) / 4 s} d y\right\} \\
& \leqslant\left|b^{*}\right|\left\{1+\left|b_{0}\right|(\ell-k) e^{\left|b_{0} \alpha\right| / 2+b_{0} b^{*} / 2}\right\}:=\left|b^{*}\right| C_{1}
\end{aligned}
$$


where $C_{1}=1+\left|b_{0}\right|(\ell-k) e^{\left|b_{0} \alpha\right| / 2+b_{0} b^{*} / 2}$. Hence, $\dot{b}(s)=O(1) s^{-3 / 2} e^{-\beta s}$ for $s \geqslant 1$.

Once we have the upper bound of $|\dot{b}|$, we can study the asymptotic behavior of the right-hand side of (8.2) as $s \rightarrow \infty$. First, for each $s \geqslant 1, t \in[0, s]$, and $y \in\left[b_{0}, 0\right]$,

$$
\delta(s, t)=O(1) s^{-1}, \quad \delta_{1}(s, y)=O(1) s^{-1}, \quad \delta_{2}(s, t)=O(1) s^{-1} .
$$

Next note that

$$
\zeta(t)=O(1) \dot{b}(t) e^{\beta t}=O(1) t^{-3 / 2} \quad \text { for } t \in[1, \infty)
$$

and $\zeta(t)=O(1) \dot{b}(t)$ for $t \in[0,1]$. Hence, for $s \geqslant 1$,

$$
\begin{aligned}
\int_{s}^{\infty} \zeta(t) d t & =O(1) \int_{s}^{\infty} t^{-3 / 2} d t=O(1) s^{-1 / 2} \\
\int_{s / 2}^{s} \zeta(t)\left(1-\frac{t}{s}\right)^{-\frac{1}{2}} d t & =O(1) \int_{s / 2}^{s} \frac{t^{-3 / 2} \sqrt{s}}{\sqrt{s-t}} d t=\frac{O(1)}{\sqrt{s}} \int_{1 / 2}^{1} \frac{\theta^{-3 / 2}}{\sqrt{1-\theta}} d \theta=\frac{O(1)}{\sqrt{s}} \\
\int_{0}^{s / 2} \frac{t}{s}\left(1+\frac{t}{s} \zeta(t) d t\right. & =\frac{O(1)}{s} \int_{0}^{1 / 2} \dot{b}(t) d t+\frac{O(1)}{s} \int_{1 / 2}^{s / 2} t^{-1 / 2} d t=\frac{O(1)}{\sqrt{s}} \\
\int_{0}^{s / 2} \frac{t+1}{s} \zeta(t) d t & =\frac{O(1)}{s} \int_{0}^{1 / 2} \dot{b}(t) d t+O(1) \int_{1 / 2}^{s / 2} \frac{t}{s} t^{-3 / 2}\left(1+\frac{t}{s}\right) d t=\frac{O(1)}{\sqrt{s}}
\end{aligned}
$$

Thus, using $(1-t / s)^{-1 / 2}=1+O(1) t / s$ for $t \in[0, s / 2]$ we obtain from (8.2) that

$$
\begin{aligned}
& \frac{\dot{b}(s)\left[\ell e^{b(s)}-k\right] s}{\Gamma(b(s), s)}=\int_{b_{0}}^{0} y A_{\infty}(y) e^{\delta_{1}(s, y)} d y+\int_{0}^{s}[t \delta(s, t)-b(t)] \zeta(t)\left(1-\frac{t}{s}\right)^{-1 / 2} e^{\delta_{2}(s, t)} d t \\
= & \int_{b_{0}}^{0} y A_{\infty}(y)\left(1+\frac{O(1)}{s}\right) d y+\int_{0}^{s}\left[t\left(1+\frac{O(1)}{s}\right)-b(t)\right] \zeta(t)\left(1-\frac{t}{s}\right)^{-1 / 2}\left(1+\frac{O(1)}{s}\right) d t \\
= & \int_{b_{0}}^{0} y A_{\infty}(y) d y+\left(1+\frac{O(1)}{s}\right) \int_{0}^{s} \frac{t}{s} \zeta(t)\left(1-\frac{t}{s}\right)^{-1 / 2} d t \\
& -\left(1+\frac{O(1)}{s}\right) \int_{o}^{s} b(t) \zeta(t)\left(1-\frac{t}{s}\right)^{-1 / 2} d t+\frac{O(1)}{s} \\
= & \left(1+\frac{O(1)}{s}\right) \int_{s / 2}^{s} \zeta(1-t / s)^{-1 / 2} d t+\left(1+\frac{O(1)}{s}\right) \int_{0}^{s / 2} \frac{t}{s} \zeta(t)\left(1+\frac{t}{s}\right) d t+\frac{O(1)}{s} \\
& -\left(1+\frac{O(1)}{s}\right) \int_{s / 2}^{s} b(t) \zeta(t)\left(1-\frac{t}{s}\right)^{-1 / 2} d t+\left(1+\frac{O(1)}{s}\right) \int_{0}^{s / 2} b(t) \zeta(t) d t+\int_{b_{0}}^{0} y A_{\infty}(y) d y \\
= & \left(1+\frac{O(1)}{s}\right) O(1) \int_{0}^{s / 2} b(t) \zeta(t) d t+\int_{b_{0}}^{0} y A_{\infty}(y) d y+\frac{O(1)}{\sqrt{s}} \\
= & -\left(1+\frac{O(1)}{s}\right) \int_{0}^{\infty} b(t) \zeta(t) d t-O(1) \int_{s / 2}^{\infty} \zeta(t) d t+\frac{O(1)}{\sqrt{s}}
\end{aligned}
$$


which implies

$$
\frac{\dot{b}(s)\left[\ell e^{b(s)}-k\right] s}{\Gamma(b(s), s)}=\int_{b_{0}}^{0} y A_{\infty}(y) d y-\int_{0}^{\infty} b(t) \zeta(t) d t+\frac{O(1)}{\sqrt{s}}:=m_{1}+\frac{O(1)}{\sqrt{s}} .
$$

Noting that $b(s)=b^{*}+O(1) e^{-\beta s}$, one obtains

$$
\dot{b}(s)=-\left[m_{1}+O(1) s^{-1 / 2}\right] \frac{\Gamma(b(s), s)}{\left[\ell e^{b(s)}-k\right] s}=-\left[m \beta+O(1) s^{-1 / 2}\right] s^{-\frac{3}{2}} e^{-\beta s}
$$

and after integration, $b(s)=b^{*}+\left[m+O(1) s^{-1 / 2}\right] s^{-3 / 2} e^{-\beta s}$, where $m=m_{1} e^{-\alpha b^{*} / 2} /(\sqrt{4 \pi}[k-$ $\left.\left.\ell e^{b^{*}}\right] \beta\right)$.

To see that $m$ is positive, we let $s \rightarrow \infty$ in (8.1) to obtain the identity $1+\int_{b_{0}}^{0} A_{\infty}(y) d y-$ $\int_{0}^{\infty} \zeta(t) d t=0$. Adding a multiple of $-b_{0}$ of this identity to the defining equation of $m_{1}$ we find that

$$
m_{1}=-b_{0}+\int_{b_{0}}^{0}\left[y-b_{0}\right] A_{\infty}(y) d y+\int_{0}^{\infty}\left[b_{0}-b(t)\right] \zeta(t) d t>0 .
$$

This completes the proof of Theorem 8.1.

Proof of Theorem 8.2. Note that the function $q(x, s):=p_{s}(x, s) e^{\alpha x / 2+\beta s}$ satisfies

$$
\begin{aligned}
& q_{s}-q_{x x}=0 \quad \text { in } \quad Q_{b}:=\{(x, s) \mid x>b(s), s>0\} \\
& q(b(s), s)=0 \quad \forall s>0 .
\end{aligned}
$$

Since $p \geqslant p_{0}$ implies $q(\cdot, 0)=p_{s}(\cdot, 0) \geqslant 0$, so by the maximum principle and Hopf's Lemma, $q>0$ in $Q_{b}$ (see chapter 3 for the details of a rigorous derivation). We shall construct comparison functions to estimate the upper and lower bound of $q$.

Upper bound. Fix an arbitrary $T>0$. Let $\bar{q}$ be the solution of the problem

$$
\begin{array}{ll}
\bar{q}_{s}-\bar{q}_{x x}=0 \quad \text { in }[b(T), \infty) \times(0, T], \\
\bar{q}=0, & \text { on }\{b(T)\} \times[0, T], \\
\bar{q}=q, & \text { on }[b(T), \infty) \times\{0\} .
\end{array}
$$

Then by comparison, $q \leqslant \bar{q}$ on $[b(T), \infty) \times[0, T]$. In particular, for $x \geqslant b(T)$,

$$
q(x, T) \leqslant \bar{q}(x, T)=\int_{b(T)}^{\infty} \frac{e^{-(x-y)^{2} /(4 T)}-e^{-(x+y-2 b(T))^{2} /(4 T)}}{\sqrt{4 \pi T}} q(y, 0) d y .
$$


Using $e^{-a}-e^{-b} \leqslant e^{-a}(b-a)$ for $0 \leqslant a \leqslant b$, one obtains

$$
\begin{aligned}
& e^{-(x-y)^{2} /(4 T)}-e^{-(x+y-2 b(T))^{2} /(4 T)} \\
= & e^{-(x-y)^{2} / 4 T}\left[(x+y-2 b(T))^{2}-(x-y)^{2}\right] / 4 T \\
= & e^{-(x-y)^{2} / 4 T}(x-b(T))(y-b(T)) / T .
\end{aligned}
$$

Applying this in (8.9) and noting the fact $q(x, 0)=\delta(x)+e^{\alpha x / 2}\left[\ell e^{x}-k\right] \chi_{\left[b_{0}, 0\right]}(x)$, where $\delta$ is the Dirac measure and $\chi_{A}$ is the characteristic function of the set $A$, one then obtains, for $x \geqslant b(T)$,

$$
\begin{aligned}
q(x, T) & \leqslant \int_{b(T)}^{\infty} \frac{[x-b(T)][y-b(T)] e^{-(x-y)^{2} /(4 T)}}{\sqrt{4 \pi T^{3}}} q(y, 0) d y \\
& =\frac{(x-b(T)) e^{-x^{2} /(4 T)}}{\sqrt{4 \pi T^{3}}}\left\{-b(T)+\int_{b_{0}}^{0}(y-b(T))\left(\ell e^{y}-k\right) e^{-y^{2} /(4 T)+\alpha y / 2+x y /(2 T)} d y\right\} \\
& \leqslant \frac{(x-b(T)) e^{-x^{2} /(4 T)}|b(T)|}{\sqrt{4 \pi T^{3}}}\left\{1+\left|b_{0}\right|(\ell-k) e^{b^{*} b_{0} /(2 T)+\left|\alpha b_{0}\right| / 2}\right\} .
\end{aligned}
$$

For $s \geqslant 1$, replacing $T$ by $s$ yields

$$
\begin{aligned}
p_{s}(x, s) & =q(x, s) e^{-\alpha x / 2-\beta s} \\
& \leqslant|b(s)| \frac{(x-b(s)) e^{-x^{2} / 4 s}}{s \sqrt{4 \pi s}}\left\{1+\left|b_{0}\right|(\ell-k) e^{\left(b^{*}+\alpha\right) b_{0} / 2}\right\} \\
& \leqslant|b(s)|[x-b(s)]^{+} s^{-1} \Gamma(x, s) C_{1}
\end{aligned}
$$

where $C_{1}=1+\left|b_{0}\right|(\ell-k) e^{\left(b^{*}+\alpha\right) b_{0} / 2}$. Consequently, noting that $p_{s}(x, s)=0$ for $x<b(s)$,

$$
\left\|p_{s}(\cdot, s)\right\|_{L^{\infty}(\mathbb{R})} \leqslant C_{1}\left|b^{*}\right| \max _{x>b^{*}}\left\{\left(x-b^{*}\right) s^{-1} \Gamma(x, s)\right\} \leqslant C_{2} \rho(s)
$$

To compute $\max _{x>b^{*}}\left\{\left(x-b^{*}\right) s^{-1} \Gamma(x, s)\right\}$, for each $s$, define

$$
f(x):=\left(x-b^{*}\right) s^{-1} \Gamma(x, s)=\frac{e^{-\beta s}}{\sqrt{4 \pi s^{3}}}\left(x-b^{*}\right) e^{-x^{2} / 4 s+\alpha x / 2}, \quad \text { in } x>b^{*} .
$$

Then one has

$$
f^{\prime}(x)=\frac{e^{-\beta s} e^{-x^{2} / 4 s+\alpha x / 2}}{\sqrt{4 \pi s^{3}}}\left[\frac{2 s-\left(x-b^{*}\right)(x-2 \alpha s)}{2 s}\right]
$$


which implies that $f(x)$ reach its maximum at its one critical point $x_{0}$, where

$$
x_{0}:=\frac{\left(b^{*}+2 \alpha s\right)+\sqrt{\left(b^{*}-2 \alpha s\right)^{2}+8 s}}{2} .
$$

Therefore $\left\|p_{s}(\cdot, s)\right\|_{L^{\infty}(\mathbb{R})} \leqslant C_{1}\left|b^{*}\right| f\left(x_{0}\right)$.

To estimate $f\left(x_{0}\right)=\left(x_{0}-b^{*}\right) \exp \left(-x_{0}^{2} / 4 s+\alpha x_{0} / 2\right) e^{-\beta s} / \sqrt{4 \pi s^{3}}$ as $s \nearrow \infty$, note that the quantity $\left(x_{0}-b^{*}\right) \exp \left(-x_{0}^{2} / 4 s+\alpha x_{0} / 2\right)$ is increasing as $s$ increasing, hence

$$
\left(x_{0}-b^{*}\right) \exp \left(-\frac{x_{0}^{2}}{4 s}+\frac{\alpha x_{0}}{2}\right) \leq \lim _{s \rightarrow \infty}\left(x_{0}-b^{*}\right) \exp \left(-\frac{x_{0}^{2}}{4 s}+\frac{\alpha x_{0}}{2}\right) .
$$

When $\alpha<0$, as $s \nearrow \infty$

$$
x_{0}=\frac{8 s\left(\alpha b^{*}-1\right)}{\left(b^{*}+2 \alpha s\right)-\sqrt{\left(b^{*}-2 \alpha s\right)^{2}+8 s}} \rightarrow-\frac{\alpha b^{*}-1}{\alpha}:=x_{0}^{*} .
$$

Hence $C_{1}\left|b^{*}\right| f\left(x_{0}\right) \leqslant C_{2} s^{-3 / 2} e^{-\beta s}$. When $\alpha=0, x_{0} / \sqrt{s} \rightarrow \sqrt{8}$ and $f\left(x_{0}\right) \rightarrow s^{-1} e^{-\beta s} / e^{4} \sqrt{4 \pi}$, when $\alpha>0, x_{0} / s \rightarrow 2 \alpha$ and

$$
f\left(x_{0}\right)=\frac{s^{-1} e^{-\beta s}}{\sqrt{4 \pi}} \frac{\left(x_{0}-b^{*}\right)}{s} e^{-\frac{x_{0}}{4 s}\left(x_{0}-2 \alpha x_{0}\right)}=\frac{s^{-1} e^{-\beta s}}{\sqrt{4 \pi}} \frac{\left(x_{0}-b^{*}\right)}{s} e^{-\frac{x_{0}}{4 s} \frac{2 s}{x_{0}-b^{*}}}
$$

which implies $C_{1}\left|b^{*}\right| f\left(x_{0}\right) \leqslant C_{2} s^{-1 / 2} e^{-\beta s}$. Notice that all constants depend only on $k$ and $\ell$. In addition, for $s \geqslant 1$,

$$
\left\|p(\cdot, s)-p^{*}(\cdot)\right\|_{L^{\infty}(\mathbb{R})} \leqslant \int_{s}^{\infty}\left\|p_{s}(\cdot, t)\right\|_{L^{\infty}(\mathbb{R})} d t \leqslant \int_{s}^{\infty} C_{2} \rho(t) d t \leqslant \frac{C_{2} \rho(s)}{k} .
$$

where the last inequality requires

$$
\int_{s}^{\infty} t^{-n} e^{-\beta t} d t \leqslant t^{-n} \int_{s}^{\infty} e^{-\beta s} d t=\frac{s^{-n} e^{-\beta s}}{\beta}
$$

Lower bound. Fix an arbitrary $\varepsilon>0$. Let $\underline{q}$ be the solution of

$$
\underline{\mathrm{q}}_{s}-\underline{\mathrm{q}}_{x x}=0 \text { in }[0, \infty) \times(\varepsilon, \infty), \quad \underline{\mathrm{q}}=0 \text { on }\{0\} \times[\varepsilon, \infty), \quad \underline{\mathrm{q}}=q \text { on }[0, \infty) \times\{\varepsilon\} .
$$


Then by comparison, $q>\underline{\mathrm{q}}$ on $[0, \infty) \times(\varepsilon, \infty)$. Thus, for $s>0$ and $x>0$,

$$
\begin{aligned}
q(x, s+\varepsilon) & \geqslant \underline{\mathrm{q}}(x, s+\varepsilon)=\int_{0}^{\infty} \frac{e^{-(x-y)^{2} /(4 s)}-e^{-(x+y)^{2} /(4 s)}}{\sqrt{4 \pi s}} q(y, \varepsilon) d y \\
& =\frac{e^{-x^{2} /(4 s)}}{\sqrt{4 \pi s}} \int_{0}^{\infty} e^{-y^{2} /(4 s)}\left(e^{x y /(2 s)}-e^{-x y /(2 s)}\right) q(y, \varepsilon) d y .
\end{aligned}
$$

Using $e^{z}-e^{-z} \geqslant 2 z$ for $z \geqslant 0$ we then obtain, when $x>0$ and $s>1$

$$
q(x, s+\varepsilon) \geqslant \frac{x e^{-x^{2} /(4 s)}}{\sqrt{4 \pi s^{3}}} \int_{0}^{\infty} y q(y, \varepsilon) e^{-y^{2}} d y .
$$

Setting $C_{\varepsilon}=\int_{0}^{\infty} y e^{-y^{2}} p_{s}(y, \varepsilon) e^{\alpha y / 2} d y$ we obtain for $x>0$ and $s>1$,

$$
p_{s}(x, s+\varepsilon)=q(x, s+\varepsilon) e^{-\alpha x / 2-\beta(s+\varepsilon)} \geqslant x s^{-1} \Gamma(x, s) C_{\varepsilon},
$$

which yields

$$
\begin{aligned}
p^{*}(x)-p(x, s+\varepsilon) & =\int_{s}^{\infty} p_{s}(x, t+\varepsilon) d t \\
& \geqslant \int_{s}^{s+1} x t^{-1} \Gamma(x, t) d t C_{\varepsilon} \geqslant x s^{-1} \Gamma(x, s) \int_{0}^{1} \frac{C_{\varepsilon} e^{-\beta \hat{t}}}{\sqrt{8}} d \hat{t} .
\end{aligned}
$$

Fixing $\varepsilon=1 / 2$ and finding the maximum of $x s^{-1} \Gamma(x, s)$ for $x \in(0, \infty)$ we then obtain

$$
\left\|p_{s}(\cdot, s)\right\|_{L^{\infty}(\mathbb{R})} \geqslant C_{3} \rho(s), \quad\left\|p(\cdot, s)-p^{*}(\cdot)\right\|_{L^{\infty}(\mathbb{R})} \geqslant C_{3} \rho(s)
$$

where $C_{3}$ is a positive constant depending only on $k$ and $\ell$. This completes the proof.

Proof of Theorem 8.3. From (4.5), one derives, for every $x \in \mathbb{R}$ and $s>0$,

$$
p_{s}(x, s)=\Gamma(x, s)\left\{1+I_{1}(x, s)-I_{2}(x, s)\right\}
$$

where

$$
\begin{aligned}
I_{1}(x, s) & :=\int_{b_{0}}^{0}\left[\ell e^{y}-k\right] \frac{\Gamma(x-y, s)}{\Gamma(x, s)} d y=\int_{b_{0}}^{0}\left[\ell e^{y}-k\right] e^{\alpha y / 2+x y /(2 s)-y^{2} /(4 s)}, \\
I_{2}(x, s) & :=\int_{0}^{s} \dot{b}(t)\left[\ell e^{b(t)}-k\right] \frac{\Gamma(x-b(t), s-t)}{\Gamma(x, s)} d t .
\end{aligned}
$$

Note that when $s \geqslant 1$ and $x \geqslant b^{*}, I_{2}$ is positive and

$$
I_{1}(x, s) \leqslant\left|b_{0}\right|(\ell-k) e^{\left(\alpha+b^{*}\right) b_{0} / 2} .
$$


Noting that $p_{s}(x, s)=0$ for $x \leqslant b(s)$ and $p_{x}(x, s)>0$ for $x>b(s)$, by the maximum principle from chapter 3 , we hence have the bound

$$
0 \leqslant p_{s}(x, s) \leqslant \Gamma(x, s)\left(1+(\ell-k) e^{\left(\alpha+b^{*}\right) b_{0} / 2}\right):=C_{1} \Gamma(x, s) \quad \forall x \in \mathbb{R}, s \geqslant 1
$$

The case $\boldsymbol{\alpha}<\mathbf{0}$. Define $\eta:=(x+\alpha s) /(2 s)$. For $s \geqslant 1$,

$$
\begin{aligned}
I_{1}(x, s) & =\int_{b_{0}}^{0}\left[\ell e^{y}-k\right] e^{-y^{2} /(4 s)+\eta y} d y \\
& =e^{-\hat{y}^{2} /(4 s)} \int_{b_{0}}^{0}\left[\ell e^{y}-k\right] e^{\eta y} d y=\left[1+\frac{O(1)}{s}\right] \phi_{1}(\eta)
\end{aligned}
$$

where $\hat{y} \in\left[b_{0}, 0\right]$ and $\phi_{1} \in C^{\infty}(\mathbb{R})$ is defined by

$$
\phi_{1}(\eta)=\int_{b_{0}}^{0}\left[\ell e^{y}-k\right] e^{y \eta} d y \quad \forall \eta \in \mathbb{R} .
$$

We write $I_{2}(x, s)=\int_{0}^{s} B_{0}(s, t) e^{w} d t$ where

$$
\begin{aligned}
& B_{0}(s, t):=\zeta(t)\left(1-\frac{t}{s}\right)^{-\frac{1}{2}}, \\
& w:=-\frac{t x^{2}}{4 s(s-t)}+\frac{x b(t)}{2(s-t)}-\frac{b^{2}(t)}{4(s-t)} .
\end{aligned}
$$

First consider the case $|\eta| \leqslant|\alpha| / 4$. Then

$$
\frac{x}{2 s}=\eta-\frac{\alpha}{2} \geqslant-\frac{|\alpha|}{4}-\frac{\alpha}{2} \geqslant-\frac{|\alpha|}{2},
$$

which implies

$$
w \leqslant-\frac{t x^{2}}{4 s(s-t)} \leqslant-\frac{t x^{2}}{4 s^{2}} \leqslant-\frac{\alpha^{2} t}{16} .
$$

Define $\hat{s}:=\min \left\{\frac{s}{2}, \frac{16}{\alpha^{2}} \ln s\right\}$, then for $t>\hat{s}$,

$$
\begin{aligned}
\int_{\hat{s}}^{s} B_{0}(s, t) e^{w} d t & \leqslant e^{-\alpha^{2} \hat{s} / 16} \int_{0}^{s} B_{0}(s, t) d t \\
& \leqslant \frac{O(1)}{s}\left\{\int_{0}^{1 / 2} \dot{b}(t) d t+\int_{s / 2}^{s}\left(1-\frac{t}{s}\right)^{-1 / 2} d t\right\}=\frac{O(1)}{s} .
\end{aligned}
$$


When $t \in[0, \hat{s}]$,

$$
\begin{aligned}
w & =\left\{-\frac{t x^{2}}{4 s^{2}}+\frac{x b(t)}{2 s}-\frac{b^{2}(t)}{4 s}\right\}\left(1-\frac{t}{s}\right)^{-1} \\
& =\left\{\left(\eta-\frac{\alpha}{2}\right)^{2} t+\left(\eta-\frac{\alpha}{2}\right) b(t)+\frac{O(1)}{s}\right\}\left(1+\frac{t}{s}+\ldots\right) \\
& =\left(\eta-\frac{\alpha}{2}\right)^{2} t+\left(\eta-\frac{\alpha}{2}\right) b(t)+O(1) \frac{t^{2}+1}{s} .
\end{aligned}
$$

Thus,

$$
\begin{aligned}
I_{2}(x, s) & =\int_{0}^{s} B_{0}(s, t) e^{w} d t=\int_{\hat{s}}^{s} B_{0}(s, t) e^{w} d t+\int_{0}^{\hat{s}} B_{0}(s, t) e^{w} d t \\
& =\frac{O(1)}{s}+\int_{0}^{\hat{s}} \zeta(t)\left[1+\frac{O(1) t}{s}\right] e^{-(\eta-\alpha / 2)^{2} t+(\eta-\alpha / 2) b(t)}\left[1+\frac{O(1)\left(t^{2}+1\right)}{s}\right] d t \\
& =\int_{0}^{\hat{s}} \zeta(t) e^{-[\eta-\alpha / 2]^{2} t+[\eta-\alpha / 2] b(t)} d t+\frac{O(1)}{s}=\phi_{2}(\eta)+\frac{O(1)}{s}
\end{aligned}
$$

where $\phi_{2}$ is defined by

$$
\phi_{2}(\eta):=\int_{0}^{\infty} \dot{b}(t)\left[\ell e^{b(t)}-k\right] e^{\left[\beta-(\eta-\alpha / 2)^{2}\right] t+\eta b(t)} d t
$$

Since $\dot{b}(t)=O(1) t^{-3 / 2} e^{-\beta t}$ for $t \geqslant 1$ and $\dot{b}(t) d t=d b(t)$ is a bounded measure on [0,1] with integrand being continuous in $t$ and analytic in $\eta, \phi_{2} \in C(\mathbb{R}) \cap C^{\infty}((-\infty, \alpha / 2) \cup(\alpha / 2, \infty))$.

In summary, setting $\phi(\eta)=1+\phi_{1}(\eta)-\phi_{2}(\eta)$ we have, when $|\eta| \leqslant|\alpha| / 4$ and $s \geqslant 1$,

$$
\frac{p_{s}(x, s)}{\Gamma(x, s)}=1+I_{1}-I_{2}=\phi(\eta)+O(1) s^{-1}=\phi(0)+\phi^{\prime}(0) \eta+O(1) \eta^{2}+O(1) s^{-1}
$$

Note that when $|\eta|>|\alpha| / 4$, both $\eta$ and $s^{-1}$ are $O(1)$, so the last expansion is still valid since it was established earlier that $p_{s} / \Gamma=O(1)$. The assertion of Theorem 8.3 for $p_{s} / \rho(s)$ with $\alpha<0$ thus follows with $c_{1}:=\phi(0) / \sqrt{4 \pi}$ and $c_{2}:=\phi^{\prime}(0) / \sqrt{4 \pi}$. From the inequality (8.10), one has

$$
\phi(\eta)+O(1)=\frac{p_{s}(x, s)}{\Gamma(x, s)} \geqslant C_{\varepsilon} \frac{x}{s}
$$

which implies that $c_{1}>0$ since $x=-\alpha s>0$ when $\eta=0$. 
The case $\boldsymbol{\alpha} \geqslant \mathbf{0}$. This implies that $k \geqslant \ell+1$ so $b_{0}=0$. Using $p_{s}(b(s), s)=0$ we obtain from (8.11) that

$$
\frac{p_{s}(x, s)}{\Gamma(x, s)}=\frac{p_{s}(x, s)}{\Gamma(x, s)}-\frac{p_{s}(b(s), s)}{\Gamma(b(s), s)}=\int_{0}^{s} B(s, t)\left\{1-e^{u}\right\} d t
$$

where $B(s, t)$ is the same as before and

$$
\begin{aligned}
u & =\frac{x^{2}-b^{2}(s)}{4 s}-\frac{[x-b(t)]^{2}-[b(s)-b(t)]^{2}}{4(s-t)} \\
& =(x-b(s))\left\{\frac{b(t)}{2(s-t)}-\frac{t[x+b(s)]}{4 s(s-t)}\right\}
\end{aligned}
$$

Hence, $u<0$, when $x \geqslant-b(s)>b(s)$. From above equality, one has

$$
\begin{aligned}
u & =-\frac{t[x-b(s)]^{2}}{4 s(s-t)}+\frac{[x-b(s)]}{2 s}\left\{\frac{b(t)}{2(s-t)}-\frac{2 t b(s)]}{2 s(s-t)}\right\} \\
& =-\frac{t[x-b(s)]^{2}}{4 s(s-t)}+\frac{[x-b(s)]}{2 s}\{b(t)-t \delta(s, t)\}
\end{aligned}
$$

So if $|x|<-b(s)$, noting $\delta(s, t)<0$ and $\delta(s, t)=O(1) / s$, one has

$$
\begin{aligned}
u & \leqslant \frac{[x-b(s)]}{2 s}\{b(t)-t \delta(s, t)\} \leqslant \frac{[x-b(s)]}{2 s} t[-\delta(s, t)] \\
& \leqslant \frac{[-2 b(s)]}{2 s} t[-\delta(s, t)] \leqslant|b(s) \delta(s, t)|=\frac{O(1)}{s}
\end{aligned}
$$

Hence, $u \leqslant O(1) / s$ for all $x \geqslant b(s)$.

Denoting $z=[x-b(s)] / \sqrt{4 s}$, we can write

$$
u=u_{1}+u_{2}, \quad u_{1}:=-\frac{t z^{2}}{s-t}, \quad u_{2}:=\frac{z}{\sqrt{s}}[b(t)-t \delta(s, t)]
$$

We first consider the case when $z \in[0, \sqrt{s}]$, i.e., $b(s)<x<b(s)+2 s$. We write

$$
\begin{aligned}
\frac{p_{s}(x, s)}{\Gamma(x, s)}=\int_{0}^{s} & \left\{\left[B-B_{0}\right]\left[1-e^{u}\right]+B_{0}\left[1-e^{u_{2}}\right]\right. \\
& \left.+B_{0}\left[1-e^{u_{1}}\right]-B_{0}\left[1-e^{u_{2}}\right]\left[1-e^{u_{1}}\right]\right\} d t
\end{aligned}
$$

To estimate (8.12), We first claim that

$$
\int_{0}^{s} B_{0}\left[1-e^{u}\right] d t=O(1) z / \sqrt{s}
$$


Recalling that $\delta(s, t)=O(1) s^{-1}$, we have

$$
\begin{aligned}
\int_{0}^{s} B_{0}(s, t)\left[1-e^{u_{2}}\right] d t & =\int_{0}^{s} B_{0}\left[-u_{2}-u_{2}^{2}-\ldots\right] \\
& =\int_{0}^{s} B_{0}(s, t)\left\{-\frac{z[b(t)-t \delta(s, t)]}{\sqrt{s}}+O(1) \frac{z^{2}}{s}\right\} d t \\
& =-\frac{z}{\sqrt{s}} \int_{0}^{\infty} \zeta(t) b(t) d t+O(1) \frac{z+z^{2}}{s} \\
& =\frac{m_{1} z}{\sqrt{s}}+O(1) \frac{z+z^{2}}{s},
\end{aligned}
$$

where $m_{1}=-\int_{0}^{\infty} b(t) \zeta(t) d t$ and the third equality is obtained by using (8.3)-(8.5).

To evaluate $\int_{0}^{s} B_{0}(s, t)\left[1-e^{u_{1}}\right] d t$, we use the expansion

$$
\zeta(t)=\dot{b}(t)\left[\ell e^{b(t)}-k\right] e^{\alpha b(t) / 2+\beta t}=\hat{m} t^{-3 / 2}+O\left(t^{-2}\right)
$$

where $\hat{m}=m \beta\left[k-\ell e^{b^{*}}\right] e^{\alpha b^{*} / 2}=m_{1} / \sqrt{4 \pi}$. When $\zeta(t)$ is replaced by $\hat{m} t^{-3 / 2}$, the corresponding integral can be evaluated by the substitution $\hat{t}=-u_{1}=t z^{2} /(s-t)$, giving

$$
\int_{0}^{s} \hat{m} t^{-3 / 2}\left[1-e^{u_{1}}\right]\left(1-\frac{t}{s}\right)^{-1 / 2} d t=\frac{2 \sqrt{\pi} \hat{m} z}{\sqrt{s}}=\frac{m_{1} z}{\sqrt{s}} .
$$

We estimate the error of replacing $\zeta$ by $\hat{m} t^{-3 / 2}$ as follows. Taking $t_{1}=\max \{s / 2, s /(1+$ $\left.\left.z^{2}\right)\right\}$ and using $\zeta(t)=O(1) \dot{b}(t)$ for $t \in(0,1]$ and $\zeta(t)-\hat{m} t^{-3 / 2}=O(1) t^{-2}$ for $t \in[1, s]$ we obtain, when $s \geqslant 2$,

$$
\begin{gathered}
\int_{0}^{s}\left|B_{0}-\hat{m} t^{-3 / 2}\left(1-\frac{t}{s}\right)^{-1 / 2}\right|\left(1-e^{u_{1}}\right) \\
<\int_{0}^{s}\left|\zeta-\hat{m} t^{-3 / 2}\right| \min \left\{1, \frac{t z^{2}}{s-t}\right\} \frac{\sqrt{s}}{\sqrt{s-t}} d t \\
=O(1)\left\{\int_{0}^{1}\left(t^{-3 / 2}+|\dot{b}|\right) \frac{t z^{2}}{s} d t+\int_{1}^{s / 2} \frac{z^{2}}{t s} d t\right. \\
\left.+\int_{s / 2}^{t_{1}} \frac{z^{2}}{\sqrt{s} \sqrt{(s-t)^{3}}} d t+\int_{t_{1}}^{s} \frac{1}{s^{3 / 2} \sqrt{s-t}} d t\right\} \\
=O(1)\left(\frac{z^{2}}{s}+\frac{z^{2} \ln s}{s}+\frac{\sqrt{s-t_{1}}}{\sqrt{s} \sqrt{s-t_{1}}}+\frac{z^{3 / 2}}{s}\right) . \\
=O(1)\left(\frac{z}{s}+\frac{z^{2} \ln s}{s}\right)
\end{gathered}
$$


Then, since $\left|1-e^{u_{2}}\right|=O(1) z / \sqrt{s}$,

$$
\int_{0}^{s} B_{0}\left|\left[1-e^{u_{2}}\right]\right|\left[1-e^{u_{1}}\right] d t=\frac{O(1) z}{\sqrt{s}} \int_{0}^{s} B_{0}\left[1-e^{u_{1}}\right] d t=O(1) \frac{z^{2}}{s}
$$

Finally, we have the claim (8.13),

$$
\begin{aligned}
& \int_{0}^{s} B_{0}\left[1-e^{u}\right] d t \\
= & \int_{0}^{s} B_{0}\left[1-e^{u_{1}}\right]+B_{0}\left[1-e^{u_{2}}\right]-B_{0}\left[1-e^{u_{1}}\right]\left[1-e^{u_{2}}\right] d t \\
= & \frac{O(1) z}{\sqrt{s}}
\end{aligned}
$$

Using $\delta_{2}(s, t)=O(1) / s$, one obtains

$$
B-B_{0}=B_{0}\left[e^{\delta_{2}(s, t)}-1\right]=\frac{O(1)}{s} B_{0}
$$

Then noting that $u \leqslant O(1) / s$, we have for $z \geqslant 0$ and $s \geqslant 1$,

$$
\int_{0}^{s}\left[B-B_{0}\right]\left[1-e^{u}\right] d s=\frac{O(1)}{s} \int_{0}^{s} B_{0}\left|1-e^{u}\right| d t=\frac{O(1)}{s} \frac{z}{\sqrt{s}},
$$

In summary, we obtain from (8.12) that when $s \geqslant 2$ and $0 \leqslant z \leqslant \sqrt{s}$ with $z=(x-$ $b(s)) / \sqrt{4 s}$,

$$
\begin{aligned}
\frac{p_{s}(x, s)}{\Gamma(x, s)} & =\frac{z}{\sqrt{s}}\left\{2 m_{1}+\frac{O(1)}{\sqrt{s}}(1+z \ln s)\right\} \\
& =\frac{x-b(s)}{s}\left\{m_{1}+O(1)\left(s^{-1 / 2}+|x| s^{-1} \ln s\right)\right\}
\end{aligned}
$$

Since $p_{s}(x, s) / \Gamma(x, s)=O(1)$, this expansion is also valid when $z>\sqrt{s}$. This implies the assertion of Theorem 8.3 for $p_{s} / \rho(s)$ with $c_{1}=m_{1} / \sqrt{\pi}$ for the case $\alpha=0$ and $c_{1}=$ $m_{1} e^{\alpha b^{*} / 2} / \sqrt{4 \pi}$ for the case $\alpha>0$.

Finally, the asymptotic behavior for $p^{*}-p$ follows by integrating $p_{s}$ over $[s, \infty)$. It first requires observing that for $n>0(n=3 / 2$ for $\alpha \geq 0$ and $n=1 / 2$ for $\alpha<0)$

$$
\begin{aligned}
J & :=\int_{s}^{\infty} t^{-n} e^{-x^{2} /(4 t)-\beta t} \\
& =-\int_{s}^{\infty} t^{-n} \frac{d\left(e^{-x^{2} /(4 t)-\beta t}\right)}{\beta-x^{2} /\left(4 t^{2}\right)}=\frac{s^{-n} e^{-x^{2} /(4 s)-\beta s}}{\beta-x^{2} /\left(4 s^{2}\right)}-A J
\end{aligned}
$$


where

$$
A:=\frac{1}{\hat{t}}\left[\frac{n}{\beta-x^{2} /\left(4 \hat{t}^{2}\right)}+\frac{x^{2}}{2 \hat{t}^{2}} \frac{1}{\left(\beta-x^{2} / 4 \hat{t}^{2}\right)^{2}}\right], \quad \hat{t} \in[s, \infty)
$$

which implies that for a small number $\varepsilon>0$,

$$
J=\frac{1}{1+O(1 / s)} \frac{s^{-n} e^{-x^{2} /(4 s)-\beta s}}{\beta-x^{2} /\left(4 s^{2}\right)} \quad \text { if }|x|<2 \sqrt{\beta-\varepsilon} s \text { i.e., } \beta-\frac{x^{2}}{4 s^{2}}>\varepsilon
$$

(since $0<A=O(1 / s)$ for $\hat{t} \geq s,|x|<2 \sqrt{\beta-\varepsilon} s$ ).

Using the above technique, we have, for $\alpha>0$ and $x \geqslant b(s)$,

$$
p^{*}-p=e^{-\alpha x / 2} t^{-3 / 2} e^{-x^{2} / 4 s-\beta s}(x-b(s)) \frac{c_{1}+O(1)\left(s^{-1 / 2}+|x| s^{-1} \ln s\right)}{\beta-x^{2} / 4 s^{2}}-J_{0}
$$

where

$$
J_{0}:=e^{-\alpha x / 2} \int_{s}^{\infty} e^{-x^{2} / 4 t} d\left\{t^{-3 / 2}(x-b(t)) \frac{c_{1}+O(1)\left(t^{-1 / 2}+|x| t^{-1} \ln t\right)}{\beta-x^{2} / 4 s^{2}}\right\}
$$

Using Theorem 8.1, one can show that the integral $J_{0}$ tends to zero faster than $\rho(s)$ and hence can be assigned to the error terms. Hence defining $c=c_{1} \beta$ we can verify that

$$
\frac{p^{*}-p}{\rho(s)}-\Psi \rightarrow c\left(x-b^{*}\right) e^{-\alpha x / 2}
$$

Similarly, after integration and letting $s \rightarrow \infty$ for $\left(p^{*}-p\right) / \rho(s)-\Psi$, we have the assertion of the theorem with $c=c_{1} / \beta$ for $\alpha<0$ and $c=c_{1} / k$ for $\alpha<0$. For $\alpha \geq 0$, the corresponding integration by parts produces an extra integral involving $\dot{b}(t)$. This completes the proof of Theorem 8.3. 


\subsection{CONCLUSIONS AND FUTURE WORK}

This thesis addressed and provided rigorous results in three areas for the early exercise boundary of the standard American put option:

(i). in the region $0<D-r \ll 1$, the early exercise boundary is not convex near expiry. This provides a rigorous negative answer to the widely accepted belief that the early exercise boundary was always convex,

(ii). the $C^{\infty}$ regularity of the early exercise boundary, and

(iii). the behavior of the early exercise boundary near and far from expiry.

In the proof of the regularity of the boundary, we also used the Schauder theorem, regularity theory for PDEs and a bootstrap argument. In our new proof of near and far from expiry behavior of the free boundary, we provided new rigorous estimates for higher order derivatives near expiry as well as the sharpest possible estimates for the boundary and the price function far from expiry. The latter used integral equation estimates, while the former was based on blow up arguments.

Some problems which continues to be under investigation include:

(i). a rigorous proof of convergence, as well as the rate of convergence, for the numerical scheme used in the thesis,

(ii). a continuing investigation of the conjectured convexity of the early exercise boundary in the region $0<D \leq r$ and in $r^{*}<D$ for some critical value of $r^{*}$, and

(iii). an estimation of the digression from convexity in the non-convex region as well as a global analytic estimation for the location of the early exercise boundary when it is convex, relying on the near and far from expiry estimates derived in the thesis. 


\section{BIBLIOGRAPHY}

[1] C. Ahn, H. Choe \& K. Lee, A long time asymptotic behavior of the free boundary for an American put, Proceedings of AMS, March 30, 2009.

[2] F. AitSahlia \& T. Lai, Exercise boundaries and efficient approximations to American option prices and hedge parameters, J. Computational Finance, 4 (2001), 85-103.

[3] G. Barone-Adesi \& R. E. Whaley, Efficient analytic approximation of American option values, Journal of Finance, 42 (1987), 301-320.

[4] G. Barone-Adesi, \& R. Elliott, Approximations for the values of American options, Stochastic Analysis and Applications, 9 (1991), 115-131.

[5] G. Barles, J. Burdeau, \& M. Romano, \& N. Samsoen, Critical stock price near expiration, Mathematical Finance, 5 (1995), 77-95.

[6] E. Bayraktar \& Hao Xing, Analysis of the optimal exercise boundary of American option for jump diffusions, SIAM J. Math. Anal.,41(2) 825-860, 2009.

[7] A. Bensoussan, On the theory of option pricing, Acta Appl. Math. 2 (1984), 139-158.

[8] A. Bensoussan \& J.L. Lions, Application of VARIATIOnAL INEQUALities in Stochastic CONTROL, North-Holland, 1982.

[9] M. Broadie \& J. Detemple, American option valuation: New bounds, approximations and comparison of existing methods, Review of Financial Studies, 9 (1996), 1121-1250.

[10] D.S. Bunch \& H. Johnson, The American put option and its critical stock price, J. Finance, 55 (2000), 2333-2356.

[11] P. Carr, R. Jarrow, \& R. Myneni, Alternative characterization of American put option, Mathematical Finance, 2 (1992), 87-105.

[12] D. Chakraborty, Numerical study of the convexity of the exercise boundary of the American put option on a dividend-paying asset, MS. Thesis, Department of Mathematics, University of Pittsburgh, December (2008). 
[13] X. Chen, H. Cheng \& J. Chadam, Non-convexity of the optimal exercise boundary for an American put option on a dividend-paying asset, submitted to Math. Finance for publication, accepted, to appear.

[14] X. Chen, H. Cheng \& J. Chadam, Far-from-expiry behavior of the American put option on a dividend-paying Asset, Proceedings of AMS, 139(2011), 273-282.

[15] X. Chen \& J. Chadam, A mathematical analysis of the optmal boundary for American put options, SIAM J. Math. Anal., 38 (2006), 1613-1641.

[16] X. Chen \& J. Chadam Analytic and numerical approximations for the early exercise boundary for American put options, Dyn. Cont. Disc. and Impulsive Sys., 10 (2003), 649-657.

[17] X. Chen, J. Chadam, L. Jiang, \& W. Zheng, Convexity of the exercise boundary of the American put option on a zero dividend asset. Math. Finance 18 (2008), 185-197.

[18] E. Ekstrom, Convexity of the optimal stopping boundary for the American put option, J. Math. Anal. Appl. 299 (2004), 147-156.

[19] A. Friedman, Variational Principles and Free Boundary Problems, John Wiley \& Sons, New York, 1982.

[20] A. Friedman, Partial Diffferential Equations of Parabolic Type, PrenticeHall, 1964.

[21] A. Friedman, Analyticity of the free boundary for the Stefan problem, Arch. Rational Mech. Anal. 61 (1976), 97-125.

[22] M.G. Garroni \& J.-L. Menaldi, Green function for SECOnd order parabolic integro-Differential Problems, Longman Scientific \& Technical, Harlow, 1992.

[23] J. Goodman \& D. Ostrov, On the early exercise boundary of the American put option, SIAM J. Appl. Math., 62, 5, (2002), 1823-1835.

[24] J. Hull, Options, Futures and Other Derivative Securities, , 6th Ed., Prentice-Hall, New York, 2008.

[25] P. Jaillet, D. Lamberton \& B. Lapeyre, Variational inequalities and the pricing of American options, Acta Applicandae Mathematicae, 21 (1990), 263-289.

[26] L. Jiang, Existence and differentiability of the solution of a two phase Stefan problem for quasi-linear parabolic equations, Chinese Math. Acta 7 (1965), 481-496.

[27] I. Karatzas, On the pricing of American option, Appl. Math. Optim. 17 (1988) 37-60. 
[28] I. Karatzas, Application of stochastic calculus in financial economics In: J.S. Baras and V. Mirelli, ed., Recent Advances in Stochatic Calculus, Progress in Automation And Information Systems, Springer-Verlag, New York: 1990.

[29] I. Karatzas, Optimization problem in the theory of continuous trading, SIAM J. Control Optim. 27 (1989), 1221-1259.

[30] I. J. Kim, The analytic evaluation of American Options, Review of Financial Studies, 3 (1990), 547-572.

[31] R. A. Kuske \& J. B. Keller, Optimal exercise boundary for an American put option, Applied Mathematical Finance 5 (1998), 107-116.

[32] Charles Knessl, Asymptotic Analysis of the American Call Option with Dividend, Euro. Jnl of Applied Mathematics, 13(2002), pp. 587-616

[33] D. Lamberton \& M. Mikou, The critical price for the American put in an exponential Lévy model, Finance Stoch. 12 (2008), 561-581.

[34] D. Lamberton \& S. Villeneuve, Critical price near maturity for an American option on a dividend-paying stock, Ann. Appl. Probability. 13 (2003), 800-815.

[35] P. Laurence, S. Salsa, Regularity of the free boundary of an American option on several assets, Comm. on Pure and Appl. Math., 62 (2009), 969-994.

[36] L. W. MacMillan, Analytic approximation for the American put option, Advances in Future and Option Research, 1 A (1986), 119-139.

[37] H. P. Jr. McKean, Appendix: a free boundary problem for the heat equation arising from a problem in mathematical economics, Industrial Management Review, 6 ( 1965), $32-39$.

[38] R. Merton, Continuous-time Finance, Blackwell, 1992.

[39] P. Van Moerbeke, On optimal stopping and free boundary problems, Arch. Rational Mech. Anal. 60 (1976), 101-148.

[40] R. Stamicar, D. Ševčovič, \& J. Chadam, The early exercise boundary for the American put near expiry: numerical approximation, Canad. Appl. Math. Quart., 7 (1999), 427444.

[41] C. Yang, L. Jiang, \& B. Bian, Free boundary and American option in a jump-diffusion model, Euro. Jnl of Applied Mathematics 17 (2006), 95-127.

[42] P. Wilmott, J. Dewynne, \& S. Howison, The Mathematics of Financial DerivaTIVEs, Cambridge,University Press, New York, 1995. 